\title{
Exponential synchronization of complex dynamical network with mixed time-varying and hybrid coupling delays via intermittent control
}

\author{
Thongchai Botmart ${ }^{1,2^{*}}$ and Piyapong Niamsup ${ }^{2,3}$
}

"Correspondence:
thongchaib@swu.ac.th
'Department of Mathematics,
Srinakharinwirot University,
Sukhumvit 23 Road, Bangkok,
10110 , Thailand
${ }^{2}$ Centre of Excellence in
Mathematics, CHE, Si Ayutthaya
Road, Bangkok, 10400, Thailand
Full list of author information is
available at the end of the article

\begin{abstract}
In this paper, we shall investigate the problem of exponential synchronization for complex dynamical network with mixed time-varying and hybrid coupling delays, which is composed of state coupling, interval time-varying delay coupling and distributed time-varying delay coupling. The designed controller ensures that the synchronization of delayed complex dynamical network are proposed via either feedback control or intermittent feedback control. The constraint on the derivative of the time-varying delay is not required which allows the time-delay to be a fast time-varying function. We use common unitary matrices, and the problem of synchronization is transformed into the stability analysis of some linear time-varying delay systems. This is based on the construction of an improved Lyapunov-Krasovskii functional combined with the Leibniz-Newton formula and the technique of dealing with some integral terms. New synchronization criteria are derived in terms of LMIs which can be solved efficiently by standard convex optimization algorithms. Two numerical examples are included to show the effectiveness of the proposed feedback control and intermittent feedback control scheme.
\end{abstract}

Keywords: exponential synchronization; complex dynamical network; mixed time-varying delays; hybrid coupling; intermittent control

\section{Introduction}

Complex dynamical network, as an interesting subject, has been thoroughly investigated for decades. These networks show very complicated behavior and can be used to model and explain many complex systems in nature such as computer networks [1], the world wide web [2], food webs [3], cellular and metabolic networks [4], social networks [5], electrical power grids [6] etc. In general, a complex network is a large set of interconnected nodes, in which a node is a fundamental unit with specific contents. As an implicit assumption, these networks are described by the mathematical term graph. In such graphs, each vertex represents an individual element in the system, while edges represent the relations between them. Two nodes are joined by an edge if and only if they interact.

In the last decade, the synchronization of complex dynamic networks has attracted much attention of researchers in this field [7-18]. Because the synchronization of complex dynamical networks can well explain many natural phenomena observed and is one of the important dynamical mechanisms for creating order in complex dynamical networks, the

(C2014 Botmart and Niamsup; licensee Springer. This is an Open Access article distributed under the terms of the Creative Commons Attribution License (http://creativecommons.org/licenses/by/2.0), which permits unrestricted use, distribution, and reproduction in any medium, provided the original work is properly cited. 
synchronization of coupled dynamical networks has come be a focal point in the study of nonlinear science. Wang and Chen introduced a uniform dynamical network model and also investigated its synchronization [11-13]. They have shown that the synchronizability of a scale-free dynamical network is robust against random removal of nodes, and yet it is fragile to specific removal of the most highly connected nodes [12]. The authors in $[14,15]$ investigated synchronization of general complex dynamical network models with coupling delays. Li and Chen [8] considered the synchronization stability of complex dynamical network models with coupling delays for both continuous- and discretetime, and they derived some synchronization conditions for both delay-independent and delay-dependent asymptotical stabilities. By utilizing Lyapunov functional method. Wang et al. [16] introduced several synchronization criteria for both delay-independent and delay-dependent asymptotical stability. Li and Yi [17] investigated synchronization of complex networks with time-varying couplings, the stability criteria were obtained by using Lyapunov-Krasovskii function method and subspace projection method. Yue and Li [18] studied the synchronization stability of continuous and discrete complex dynamical networks with interval time-varying delays in the dynamical nodes and the coupling term simultaneously, delay-dependent synchronization stability are derived in the form of linear matrix inequalities.

It is well known that the existence of time-delay in a system may cause instability and an example of oscillations can be found in systems such as chemical engineering systems, biological modeling, electrical networks, physical networks, and many others [19-25]. The stability criteria for a system with time-delays can be classified into two categories: delay-independent and delay-dependent. Delay-independent criteria do not employ any information on the size of the delay; while delay-dependent criteria make use of such information at different levels. Delay-dependent stability conditions are generally less conservative than delay-independent ones especially when the delay is small [25]. Recently, the delay-dependent stability for interval time-varying delay was investigated in $[6,18$, 20-22]. Interval time-varying delay is a time-delay that varies in an interval in which the lower bound is not restricted to be 0 . Jiang and Han [22] considered the problem of robust $H_{\infty}$ control for uncertain linear systems with interval time-varying delay based on Lyapunov functional approach in which restriction on the differentiability of the interval time-varying delay was removed. Shao [24] presented a new delay-dependent stability criterion for linear systems with interval time-varying delay, and stability criteria are derived in terms of linear matrix inequalities without introducing any free-weighting matrices. In order to reduce further the conservatism introduced by the descriptor model transformation and bounding techniques, a free-weighting matrix method is proposed in [20,26-29]. In [18], the synchronization problem has been investigated for continuous/discrete complex dynamical networks with interval time-varying delays. Based on a piecewise analysis method and the Lyapunov functional method, some new delay-dependent synchronization criteria are derived in the form of LMIs by introducing free-weighting matrices. It will be pointed out later that some existing results require more free-weighting matrix variables than our result.

Intermittent control is one of discontinuous control and has a nonzero control width. It is an engineering approach that has been widely used in engineering fields, such as manufacturing, air-quality control, transportation, and communication in practice. However, results using intermittent control to study exponential synchronization are few. In recent 
years, several synchronization criteria for complex dynamical networks with or without time-delays via feedback control or intermittent control have been presented; see [30-41] and the references therein. Synchronization of a complex dynamical network with delayed nodes by pinning periodically intermittent control was also reported in [31]. A periodically intermittent control was applied to the complex dynamical networks with both time-varying delays dynamical nodes and time-varying delays coupling in [32, 33]. In [34], the authors investigated exponential synchronization of a complex network with nonidentical time-delayed dynamical nodes by applying open-loop control to all nodes and adding some intermittent controllers to partial nodes. The authors in [31] investigated synchronization of a general model of complex delayed dynamical networks. The periodically intermittent control scheme is introduced to drive the network to achieve synchronization. Based on the Lyapunov stability theory and pinning control method, some novel synchronization criteria for such dynamical network are derived. To the best of the authors' knowledge, the problem of exponential synchronization for a complex dynamical network with mixed time-varying delays in the network hybrid coupling and time-varying delays in the dynamical nodes has not been fully investigated yet and remains open.

In this paper, inspired by the above discussions, we shall investigate the problem of exponential synchronization for a complex dynamical network with mixed time-varying and hybrid coupling delays, which is composed of constant coupling, interval time-varying delay coupling, and distributed time-varying delay coupling. The designed controller ensures that the synchronization of a delayed complex dynamical network is proposed via either feedback control or intermittent feedback control. The constraint on the derivative of the time-varying delay is not required, which allows the time-delay to be a fast timevarying function. We use common unitary matrices, and the problem of synchronization is transformed into the stability analysis of some linear time-varying delay systems. Based on the construction of an improved Lyapunov-Krasovskii functional is combined with the Leibniz-Newton formula and the technique of dealing with some integral terms. New synchronization criteria are derived in terms of LMIs which can be solved efficiently by standard convex optimization algorithms. Two numerical examples are included to show the effectiveness of the proposed feedback control and intermittent feedback control scheme.

The organization of the remaining part is as follows. In Section 2, a class of general complex dynamical network model with mixed time-varying and hybrid coupling delays and some useful lemmas are given. In Section 3, synchronization stability in complex dynamical network with mixed time-varying and hybrid coupling delays via feedback control and intermittent feedback control are investigated. Numerical examples illustrated the obtained results are given in Section 4. The paper ends with conclusions in Section 5.

\section{Network model and mathematic preliminaries}

Consider a complex dynamical network consisting of $N$ identical coupled nodes, with each node being an $n$-dimensional dynamical system

$$
\begin{aligned}
\dot{x}_{i}(t)= & f\left(x_{i}(t), x_{i}(t-h(t)), \int_{t-k_{1}(t)}^{t} x_{i}(s) d s\right)+c_{1} \sum_{j=1}^{N} a_{i j} G_{1} x_{j}(t)+c_{2} \sum_{j=1}^{N} b_{i j} G_{2} x_{j}(t-h(t)) \\
& +c_{3} \sum_{j=1}^{N} c_{i j} G_{3} \int_{t-k_{1}(t)}^{t} x_{j}(s) d s+\mathcal{U}_{i}(t), \quad t \geq 0, i=1,2, \ldots, N \\
x_{i}(t)= & \phi_{i}(t), \quad t \in\left[-\tau_{\max }, 0\right], \tau_{\max }=\max \left\{h_{2}, d, k_{1}, k_{2}\right\}
\end{aligned}
$$


where $x_{i}(t)=\left(x_{i 1}(t), x_{i 2}(t), \ldots, x_{i n}(t)\right)^{T} \in \mathbb{R}^{n}$ is the state vector of $i$ th node; $\mathcal{U}_{i}(t) \in \mathbb{R}^{m}$ are the control input of the node $i$; the constants $c_{1}, c_{2}, c_{3}>0$ are the coupling strength; $G_{1}=$ $\left(g_{1 i j}\right)_{n \times n}, G_{2}=\left(g_{2 i j}\right)_{n \times n}, G_{3}=\left(g_{3 i j}\right)_{n \times n} \in \mathbb{R}^{n \times n}$ are constant inner-coupling matrices, if some pairs $(i, j), 1 \leq i, j \leq n$, with $g_{1 i j} \neq 0, g_{2 i j} \neq 0$, and $g_{3 i j} \neq 0$, which means two coupled nodes are linked through their $i$ th and $j$ th state variables, otherwise $g_{1 i j}=0, g_{2 i j}=0, g_{3 i j}=0$; $A=\left(a_{i j}\right)_{N \times N}, B=\left(b_{i j}\right)_{N \times N}$, and $C=\left(c_{i j}\right)_{N \times N} \in \mathbb{R}^{N \times N}$ are the outer-coupling matrices of the network, in which $a_{i j}, b_{i j}$ are defined as follows: if there are a connection between node $i$ and node $j(j \neq i)$, then $a_{i j}=a_{j i}=1, b_{i j}=b_{j i}=1, c_{i j}=c_{j i}=1$; otherwise, $a_{i j}=a_{j i}=0$, $b_{i j}=b_{j i}=0, c_{i j}=c_{j i}=0(j \neq i)$, and the diagonal elements of matrices $A, B$, and $C$ are defined by

$$
\begin{aligned}
& a_{i i}=-\sum_{j=1, i \neq j}^{N} a_{i j}=-\sum_{j=1, i \neq j}^{N} a_{j i}, \\
& b_{i i}=-\sum_{j=1, i \neq j}^{N} b_{i j}=-\sum_{j=1, i \neq j}^{N} b_{j i}, \\
& c_{i i}=-\sum_{j=1, i \neq j}^{N} c_{i j}=-\sum_{j=1, i \neq j}^{N} c_{j i}, \quad i=1,2, \ldots, N .
\end{aligned}
$$

It is assumed that network (1) is connected in the sense that there are no isolated clusters, that is, $A, B, C$ are irreducible matrices.

Definition 2.1 [18] The delayed dynamical network (1) is said to achieve asymptotical synchronization if

$$
x_{1}(t)=x_{2}(t)=\cdots=s(t) \quad \text { as } t \rightarrow \infty,
$$

where $s(t)$ is a solution of an isolated node, satisfying

$$
\dot{s}(t)=f\left(s(t), s(t-h(t)), \int_{t-k_{1}(t)}^{t} s(\theta) d \theta\right) .
$$

In order to stabilize the origin of dynamical network (1) by means of the state feedback controller $\mathcal{U}_{i}(t)$ satisfying either $(\mathrm{H} 1)$ or $(\mathrm{H} 2)$, for $i=1,2, \ldots, n$,

$(\mathrm{H} 1): \quad \mathcal{U}_{i}(t)=D_{1 i} u_{i}(t)+D_{2 i} u_{i}(t-d(t))$

$$
+D_{3 i} \int_{t-k_{2}(t)}^{t} u_{i}(s) d s, \quad \forall t \geq t_{0}
$$

$(\mathrm{H} 2): \quad \mathcal{U}_{i}(t)=\left\{\begin{aligned} D_{4 i} u_{i}(t)+D_{5 i} u_{i}(t-d(t)) & \\ \quad+D_{6 i} \int_{t-k_{2}(t)}^{t} u_{i}(s) d s, & n \omega \leq t \leq n \omega+\delta, \\ 0, & n \omega+\delta<t \leq(n+1) \omega,\end{aligned}\right.$

where $D_{j i}, j=1,2, \ldots, 6$ are given matrices of appropriate dimensions, $u_{i}(t)=K_{i}\left(x_{i}(t)-s(t)\right)$ and $K_{i}$ is a constant matrix control gain, $\omega>0$ is the control period and $\delta>0$ is called the control width (control duration) and $n$ is a non-negative integer. Then substituting it into 
dynamical network (1), it is easy to get the following:

$$
\begin{aligned}
\dot{x}_{i}(t)= & f\left(x_{i}(t), x_{i}(t-h(t)), \int_{t-k_{1}(t)}^{t} x_{i}(s) d s\right)+c_{1} \sum_{j=1}^{N} a_{i j} G_{1} x_{j}(t) \\
& +c_{2} \sum_{j=1}^{N} b_{i j} G_{2} x_{j}(t-h(t))+c_{3} \sum_{j=1}^{N} c_{i j} G_{3} \int_{t-k_{1}(t)}^{t} x_{j}(s) d s \\
& +D_{1 i} K_{i}\left(x_{i}(t)-s(t)\right)+D_{2 i} u_{i}(t-d(t))+D_{3 i} \int_{t-k_{2}(t)}^{t} u_{i}(s) d s .
\end{aligned}
$$

Namely, the dynamical network (1) is governed by the following system:

$$
\begin{aligned}
\dot{x}_{i}(t)= & f\left(x_{i}(t), x_{i}(t-h(t)), \int_{t-k_{1}(t)}^{t} x_{i}(s) d s\right)+c_{1} \sum_{j=1}^{N} a_{i j} G_{1} x_{j}(t) \\
& +c_{2} \sum_{j=1}^{N} b_{i j} G_{2} x_{j}(t-h(t))+c_{3} \sum_{j=1}^{N} c_{i j} G_{3} \int_{t-k_{1}(t)}^{t} x_{j}(s) d s \\
& +D_{4 i} K_{i}\left(x_{i}(t)-s(t)\right)+D_{5 i} u_{i}(t-d(t))+D_{6 i} \int_{t-k_{2}(t)}^{t} u_{i}(s) d s, \\
& n \omega \leq t \leq n \omega+\delta, \\
\dot{x}_{i}(t)= & f\left(x_{i}(t), x_{i}(t-h(t)), \int_{t-k_{1}(t)}^{t} x_{i}(s) d s\right)+c_{1} \sum_{j=1}^{N} a_{i j} G_{1} x_{j}(t) \\
& +c_{2} \sum_{j=1}^{N} b_{i j} G_{2} x_{j}(t-h(t))+c_{3} \sum_{j=1}^{N} c_{i j} G_{3} \int_{t-k_{1}(t)}^{t} x_{j}(s) d s, \\
& n \omega+\delta<t \leq(n+1) \omega, i=1,2, \ldots, N .
\end{aligned}
$$

It is clear that, if the zero solutions of the dynamical network (4) and (5) are globally exponentially stable, then exponential synchronization of the controlled dynamical network (1) is achieved. The time-varying delay functions $h(t), d(t), k_{1}(t)$, and $k_{2}(t)$ satisfy the conditions

$$
0 \leq h_{1} \leq h(t) \leq h_{2}, \quad 0 \leq d(t) \leq d, \quad 0 \leq k_{1}(t) \leq k_{1}, \quad 0 \leq k_{2}(t) \leq k_{2} .
$$

The initial condition function $\phi_{i}(t)$ denotes a continuous vector-valued initial function of $t \in\left[-\tau_{\max }, 0\right]$.

In this paper, we assume that $s(t)$ is an orbitally stable solution of the above system. Clearly, the stability of the synchronized states (3) of network (1) is determined by the dynamics of the isolate node, the coupling strength $c_{1}, c_{2}$, and $c_{3}$, the inner-coupling matrices $G_{1}, G_{2}$, and $G_{3}$, and the outer-coupling matrices $A, B$, and $C$.

The following lemmas are used in the proof of the main result.

Lemma 2.2 [42] Let A, B be a family of diagonalizable matrices. Then A, B is a commuting family (under multiplication) if and only if it is a simultaneously diagonalizable family. 
Lemma 2.3 [19] For any constant symmetric matrix $M \in R^{n \times n}, M=M^{T}>0,0 \leq h_{1} \leq$ $h(t) \leq h_{2}, t \geq 0$, and any differentiable vector function $x(t) \in R^{n}$, we have
(a) $\left[\int_{t-h_{1}}^{t} \dot{x}(s) d s\right]^{T} M\left[\int_{t-h_{1}}^{t} \dot{x}(s) d s\right] \leq h_{1} \int_{t-h_{1}}^{t} \dot{x}^{T}(s) M \dot{x}(s) d s$,
(b) $\left[\int_{t-h(t)}^{t-h_{1}} \dot{x}(s) d s\right]^{T} M\left[\int_{t-h(t)}^{t-h_{1}} \dot{x}(s) d s\right] \leq\left(h(t)-h_{1}\right) \int_{t-h(t)}^{t-h_{1}} \dot{x}^{T}(s) M \dot{x}(s) d s$

$$
\leq\left(h_{2}-h_{1}\right) \int_{t-h(t)}^{t-h_{1}} \dot{x}^{T}(s) M \dot{x}(s) d s .
$$

Lemma 2.4 (Cauchy inequality [19]) For any symmetric positive definite matrix $N \in M^{n \times n}$ and $x, y \in \mathbb{R}^{n}$ we have

$$
\pm 2 x^{T} y \leq x^{T} N x+y^{T} N^{-1} y
$$

\section{Synchronization of delayed complex dynamical network via delayed feedback control and intermittent control}

In this section, we shall obtain some delay-dependent exponential synchronization criteria for general complex dynamical network with discrete and distributed time-varying delays and hybrid coupling delays (1) by strict LMI approaches. Let us set

$$
\tilde{A}_{i}=J(t)+c_{1} \lambda_{1 i} G_{1}, \quad \tilde{B}_{i}=J_{h}(t)+c_{2} \lambda_{2 i} G_{2}, \quad \tilde{C}_{i}=J_{k_{1}}(t)+c_{3} \lambda_{3 i} G_{3}
$$

and

1. $J(t)=f^{\prime}\left(s(t), s(t-h(t)), \int_{t-k_{1}(t)}^{t} s(\xi) d \xi\right) \in R^{n \times n}$ is the Jacobian of $f\left(x(t), x(t-h(t)), \int_{t-k_{1}(t)}^{t} x(s) d s\right)$ at $s(t)$ with the derivative of $f\left(x(t), x(t-h(t)), \int_{t-k_{1}(t)}^{t} x(s) d s\right)$ respect to $x(t)$,

2. $J_{h}(t)=f^{\prime}\left(s(t), s(t-h(t)), \int_{t-k_{1}(t)}^{t} s(\xi) d \xi\right) \in R^{n \times n}$ is the Jacobian of $f\left(x(t), x(t-h(t)), \int_{t-k_{1}(t)}^{t} x(s) d s\right)$ at $s(t-h(t))$ with the derivative of $f\left(x(t), x(t-h(t)), \int_{t-k_{1}(t)}^{t} x(s) d s\right)$ respect to $x(t-h(t))$,

3. $J_{k_{1}}(t)=f^{\prime}\left(s(t), s(t-h(t)), \int_{t-k_{1}(t)}^{t} s(\xi) d \xi\right) \in R^{n \times n}$ is the Jacobian of $f\left(x(t), x(t-h(t)), \int_{t-k_{1}(t)}^{t} x(s) d s\right)$ at $\int_{t-k_{1}(t)}^{t} s(\xi) d \xi$ with the derivative of $f\left(x(t), x(t-h(t)), \int_{t-k_{1}(t)}^{t} x(s) d s\right)$ respect to $\int_{t-k_{1}(t)}^{t} x(s) d s$.

Lemma 3.1 Consider the hybrid coupling delays dynamical network in (1). Let $0=\lambda_{j 1}>$ $\lambda_{j 2} \geq \lambda_{j 3} \geq \cdots \geq \lambda_{j N}, j=\{1,2,3\}$, be the eigenvalues of the outer-coupling matrices $A, B$, and $C$, respectively. If the $N-1$ following $n$-dimensional linear time-varying delays differential equations are delay-dependent exponentially stable about their zero solutions:

$$
\begin{aligned}
\dot{z}_{i}(t)= & \left(\tilde{A}_{i}+D_{4 i} K_{i}\right) z_{i}(t)+\tilde{B}_{i} z_{i}(t-h(t))+\tilde{C}_{i} \int_{t-k_{1}(t)}^{t} z_{i}(s) d s \\
& +D_{5 i} K_{i} z_{i}(t-d(t))+D_{6 i} K_{i} \int_{t-k_{2}(t)}^{t} z_{i}(s) d s, \quad n \omega \leq t \leq n \omega+\delta, i=2, \ldots, N, \quad(7) \\
\dot{z}_{i}(t)= & \tilde{A}_{i} z_{i}(t)+\tilde{B}_{i} z_{i}(t-h(t))+\tilde{C}_{i} \int_{t-k_{1}(t)}^{t} z_{i}(s) d s, \quad n \omega+\delta<t \leq(n+1) \omega, i=2, \ldots, N,
\end{aligned}
$$


then the dynamical networks (5) is exponentially stable, and then exponential synchronization of the controlled dynamical networks (1) is achieved.

Proof To investigate the stability of the synchronized states (3), set

$$
e_{i}(t)=x_{i}(t)-s(t), \quad i=1,2, \ldots, N
$$

Substituting (8) into (5), for $1 \leq i \leq N$, we have

$$
\begin{aligned}
\dot{e}_{i}(t)= & f\left(x_{i}(t), x_{i}(t-h(t)), \int_{t-k_{1}(t)}^{t} x_{i}(s) d s\right)-f\left(s(t), s(t-h(t)), \int_{t-k_{1}(t)}^{t} s_{i}(\xi) d \xi\right) \\
& +c_{1} \sum_{j=1}^{N} a_{i j} G_{1} e_{j}(t)+c_{2} \sum_{j=1}^{N} b_{i j} G_{2} e_{j}(t-h(t)) \\
& +c_{3} \sum_{j=1}^{N} c_{i j} G_{3} \int_{t-k_{1}(t)}^{t} e_{j}(s) d s+D_{4 i} K_{i}\left(e_{i}(t)\right)+D_{5 i} K_{i}\left(e_{i}(t-d(t))\right) \\
& +D_{6 i} K_{i} \int_{t-k_{2}(t)}^{t} e_{j}(s) d s, \quad n \omega \leq t \leq n \omega+\delta, \\
\dot{e}_{i}(t)= & f\left(x_{i}(t), x_{i}(t-h(t)), \int_{t-k_{1}(t)}^{t} x_{i}(s) d s\right)-f\left(s(t), s(t-h(t)), \int_{t-k_{1}(t)}^{t} s_{i}(\xi) d \xi\right) \\
& +c_{1} \sum_{j=1}^{N} a_{i j} G_{1} e_{j}(t)+c_{2} \sum_{j=1}^{N} b_{i j} G_{2} e_{j}(t-h(t)) \\
& +c_{3} \sum_{j=1}^{N} c_{i j} G_{3} \int_{t-k_{1}(t)}^{t} e_{j}(s) d s, \quad n \omega+\delta<t \leq(n+1) \omega, i=1,2, \ldots, N .
\end{aligned}
$$

Since $f(\cdot)$ is continuous differentiable, it is easy to know that the origin of the nonlinear system (9) is an asymptotically stable equilibrium point if it is an asymptotically stable equilibrium point of the following linear time-varying delays systems:

$$
\begin{aligned}
\dot{e}_{i}(t)= & J(t) e_{i}(t)+J_{h}(t) e_{i}(t-h(t))+J_{k_{1}}(t) \int_{t-k_{1}(t)}^{t} e_{i}(s) d s \\
& +c_{1} G_{1}\left(e_{1}(t), e_{2}(t), \ldots, e_{N}(t)\right)\left(a_{i 1}, \ldots, a_{i N}\right)^{T} \\
& +c_{2} G_{2}\left(e_{1}(t-h(t)), \ldots, e_{N}(t-h(t))\right)\left(b_{i 1}, \ldots, b_{i N}\right)^{T} \\
& +c_{3} G_{3} \int_{t-k_{1}(t)}^{t}\left(e_{1}(s), e_{2}(s), \ldots, e_{N}(s)\right)\left(c_{i 1}, \ldots, c_{i N}\right)^{T} d s \\
& +D_{4 i} K_{i} e_{i}(t)+D_{5 i} K_{i} e_{i}(t-d(t))+D_{6 i} K_{i} \int_{t-k_{2}(t)}^{t} e_{j}(s) d s, \\
& n \omega \leq t \leq n \omega+\delta, \\
\dot{e}_{i}(t)= & J(t) e_{i}(t)+J_{h}(t) e_{i}(t-h(t))+J_{k_{1}}(t) \int_{t-k_{1}(t)}^{t} e_{i}(s) d s \\
& +c_{1} G_{1}\left(e_{1}(t), e_{2}(t), \ldots, e_{N}(t)\right)\left(a_{i 1}, \ldots, a_{i N}\right)^{T} \\
& +c_{2} G_{2}\left(e_{1}(t-h(t)), \ldots, e_{N}(t-h(t))\right)\left(b_{i 1}, \ldots, b_{i N}\right)^{T}
\end{aligned}
$$




$$
\begin{aligned}
& +c_{3} G_{3} \int_{t-k_{1}(t)}^{t}\left(e_{1}(s), e_{2}(s), \ldots, e_{N}(s)\right)\left(c_{i 1}, \ldots, c_{i N}\right)^{T} d s, \\
& n \omega+\delta<t \leq(n+1) \omega .
\end{aligned}
$$

Letting $e(t)=\left(e_{1}(t), \ldots, e_{N}(t)\right) \in R^{n \times N}, e(t-h(t))=\left(e_{1}(t-h(t)), \ldots, e_{N}(t-h(t))\right) \in R^{n \times N}$, $\int_{t-k_{1}(t)}^{t} e(s) d s=\int_{t-k_{1}(t)}^{t}\left(e_{1}(s), e_{2}(s), \ldots, e_{N}(s)\right) d s \in R^{n \times N}, K=\operatorname{diag}\left\{K_{1}, K_{2}, \ldots, K_{N}\right\}$, and $D_{j}=$ $\operatorname{diag}\left\{D_{j 1}, D_{j 2}, \ldots, D_{j N}\right\}, j=\{4,5,6\}$, we have

$$
\begin{aligned}
\dot{e}(t)= & (J(t)+D K) e(t)+J_{h}(t) e(t-h(t))+J_{k_{1}}(t) \int_{t-k_{1}(t)}^{t} e(s) d s+c_{1} G_{1} e(t) A^{T} \\
& +c_{2} G_{2} e(t-h(t)) B^{T}+c_{3} G_{3} \int_{t-k_{1}(t)}^{t} e(s) C^{T} d s+D_{5} K e(t-d(t)) \\
& +D_{6} K \int_{t-k_{2}(t)}^{t} e(s) d s, \quad n \omega \leq t \leq n \omega+\delta, \\
\dot{e}(t)= & J(t) e(t)+J_{h}(t) e(t-h(t))+J_{k_{1}}(t) \int_{t-k_{1}(t)}^{t} e(s) d s+c_{1} G_{1} e(t) A^{T} \\
& +c_{2} G_{2} e(t-h(t)) B^{T}+c_{3} G_{3} \int_{t-k_{1}(t)}^{t} e(s) C^{T} d s, \quad n \omega+\delta<t \leq(n+1) \omega .
\end{aligned}
$$

Obviously, $A, B, C$ are diagonalizable. If $A, B$, and $C$ commute pairwise, i.e., $A B=B A$, then based on Lemma 2.2 , one can get a common unitary matrix $\hat{U} \in R^{N \times N}$ with $\hat{u}_{i} \in \mathbb{R}^{n}$ such that

$$
\hat{U}^{T} A \hat{U}=\Gamma_{1}, \quad \hat{U}^{T} B \hat{U}=\Gamma_{2}, \quad \hat{U}^{T} C \hat{U}=\Gamma_{3},
$$

where $\hat{U}^{T} \hat{U}=I, \Gamma_{j}=\operatorname{diag}\left\{\lambda_{1 j}, \ldots, \lambda_{N j}\right\}, j=\{1,2,3\}$. In addition, with (2) and the irreducible feature of $A, B$, and $C$ we can select with $\hat{u}_{1}=\frac{1}{\sqrt{N}}(1,1, \ldots, 1)^{T}$ such that $\lambda_{1 j}=0, j=\{1,2,3\}$. Using the nonsingular transform $e(t) \hat{U}=z(t)=\left(z_{1}(t), \ldots, z_{N}(t)\right) \in R^{N \times N}$, from (10), we have the following matrix equation:

$$
\begin{aligned}
\dot{z}(t)= & (J(t)+D K) z(t)+J_{h}(t) z(t-h(t))+J_{k_{1}}(t) \int_{t-k_{1}(t)}^{t} z(s) d s+c_{1} G_{1} z(t) \Gamma_{1} \\
& +c_{2} G_{2} z(t-h(t)) \Gamma_{2}+c_{3} G_{3} \int_{t-k(t)}^{t} z(s) \Gamma_{3} d s+D_{5} K z(t-d(t)) \\
& +D_{6} K \int_{t-k_{2}(t)}^{t} z(s) d s, \quad n \omega \leq t \leq n \omega+\delta, \\
\dot{z}(t)= & J(t) z(t)+J_{h}(t) z(t-h(t))+J_{k_{1}}(t) \int_{t-k_{1}(t)}^{t} z(s) d s+c_{1} G_{1} z(t) \Gamma_{1} \\
& +c_{2} G_{2} z(t-h(t)) \Gamma_{2}+c_{3} G_{3} \int_{t-k_{1}(t)}^{t} z(s) \Gamma_{3} d s, \quad n \omega+\delta<t \leq(n+1) \omega,
\end{aligned}
$$

that is,

$$
\begin{aligned}
\dot{z}_{i}(t)= & \left(\tilde{A}_{i}+D_{4 i} K_{i}\right) z_{i}(t)+\tilde{B}_{i} z_{i}(t-h(t))+\tilde{C}_{i} \int_{t-k_{1}(t)}^{t} z_{i}(s) d s \\
& +D_{5 i} K_{i} z_{i}(t-d(t))+D_{6 i} K_{i} \int_{t-k_{2}(t)}^{t} z_{i}(s) d s, \quad n \omega \leq t \leq n \omega+\delta,
\end{aligned}
$$




$$
\begin{gathered}
\dot{z}_{i}(t)=\tilde{A}_{1 i} z_{i}(t)+\tilde{B}_{i} z_{i}(t-h(t))+\tilde{C}_{i} \int_{t-k_{1}(t)}^{t} z_{i}(s) d s, \\
n \omega+\delta<t \leq(n+1) \omega, i=1, \ldots, N .
\end{gathered}
$$

Thus, we have transformed the stability problem of the dynamical networks (5) to the stability problem of the $N$ pieces of $n$-dimensional linear time-varying delays differential equations. Note that $\lambda_{1 k}=0$ corresponding to the synchronization of the dynamical networks (5), where the state $s(t)$ is an orbitally stable solution of the isolate node as assumed above in (3). If the following $N-1$ pieces of $n$-dimensional linear switched time-varying delays systems:

$$
\begin{aligned}
\dot{z}_{i}(t)= & \left(\tilde{A}_{i}+D_{4 i} K_{i}\right) z_{i}(t)+\tilde{B}_{i} z_{i}(t-h(t))+\tilde{C}_{i} \int_{t-k_{1}(t)}^{t} z_{i}(s) d s \\
& +D_{5 i} K_{i} z_{i}(t-d(t))+D_{6 i} K_{i} \int_{t-k_{2}(t)}^{t} z_{i}(s) d s, \quad n \omega \leq t \leq n \omega+\delta, \\
\dot{z}_{i}(t)= & \tilde{A}_{i} z_{i}(t)+\tilde{B}_{i} z_{i}(t-h(t))+\tilde{C}_{i} \int_{t-k_{1}(t)}^{t} z_{i}(s) d s, \\
n \omega & +\delta<t \leq(n+1) \omega, i=2, \ldots, N,
\end{aligned}
$$

are exponentially stable, then $e(t)$ will tend to the origin exponentially, which is equivalent to the synchronization of the dynamical networks (5) being exponentially stable. This completes the proof.

Lemma 3.2 Consider the hybrid coupling delays dynamical network in (1). Let $0=\lambda_{j 1}>$ $\lambda_{j 2} \geq \lambda_{j 3} \geq \cdots \geq \lambda_{j N}, j=\{1,2,3\}$, be the eigenvalues of the outer-coupling matrices $A, B$, and $C$, respectively. If the $N-1$ following $n$-dimensional linear time-varying delays differential equations are delay-dependent exponentially stable about their zero solutions:

$$
\begin{aligned}
\dot{z}_{i}(t)= & \left(\tilde{A}_{i}+D_{1 i} K_{i}\right) z_{i}(t)+\tilde{B}_{i} z_{i}(t-h(t))+\tilde{C}_{i} \int_{t-k_{1}(t)}^{t} z_{i}(s) d s \\
& +D_{2 i} K_{i} z_{i}(t-d(t))+D_{3 i} K_{i} \int_{t-k_{2}(t)}^{t} z_{i}(s) d s, \quad i=2, \ldots, N,
\end{aligned}
$$

then the dynamical networks (4) is exponentially stable, then exponential synchronization of the controlled dynamical networks (1) is achieved.

\subsection{Linear delayed feedback control}

Let us denote

$$
\begin{aligned}
& \left\|\phi_{i}\right\|=\left\|z_{i}(0)\right\|, \quad\left\|\varphi_{i}\right\|=\sup _{-\tau_{\max } \leq s \leq 0}\left\|z_{i}(s)\right\|, \quad K_{i}=-L_{i} P_{i}^{-1}, \\
& \gamma_{i}=\lambda_{\min }\left(P_{i}^{-1}\right), \\
& \ell_{i}=\lambda_{\max }\left(P_{i}^{-1}\right)+\left[2 h_{2} \lambda_{\max }\left(P_{i}^{-1} R_{i} P_{i}^{-1}\right)+h_{2} \lambda_{\max }\left(P_{i}^{-1} U_{i} P_{i}^{-1}\right)\right] \frac{1-e^{-2 \alpha h_{2}}}{2 \alpha} \\
& \quad+d \lambda_{\max }\left(P_{i}^{-1} L_{i}^{T} T_{i}^{-1} L_{i} P_{i}^{-1}\right) \frac{1-e^{-2 \alpha d}}{2 \alpha},
\end{aligned}
$$




$$
\begin{aligned}
\xi_{i}= & {\left[2 \lambda_{\max }\left(P_{i}^{-1} Q_{i} P_{i}^{-1}\right)+h_{2} \lambda_{\max }\left(P_{i}^{-1} R_{i} P_{i}^{-1}\right)+h_{2} \lambda_{\max }\left(P_{i}^{-1} U_{i} P_{i}^{-1}\right)\right] } \\
& \times \frac{1-e^{-2 \alpha h_{2}}}{2 \alpha}+k_{1} \lambda_{\max }\left(P_{i}^{-1} S_{i} P_{i}^{-1}\right) \frac{1-e^{-2 \alpha k_{1}}}{2 \alpha} \\
& +d \lambda_{\max }\left(P_{i}^{-1} L_{i}^{T} T_{i}^{-1} L_{i} P_{i}^{-1}\right) \frac{1-e^{-2 \alpha h_{2}}}{2 \alpha} \\
& +k_{2} \lambda_{\max }\left(P_{i}^{-1} L_{i}^{T} W_{i}^{-1} L_{i} P_{i}^{-1}\right) \frac{1-e^{-2 \alpha d}}{2 \alpha}, \\
\mathcal{N}_{i}= & \ell_{i}\left\|\phi_{i}\right\|^{2}+\xi_{i}\left\|\varphi_{i}\right\|^{2}, \\
\gamma= & \min \left\{\gamma_{i}, i=2,3, \ldots, N\right\}, \quad \mathcal{N}=\max \left\{\mathcal{N}_{i}, i=2,3, \ldots, N\right\} .
\end{aligned}
$$

Theorem 3.3 For some given scalars $0<\alpha$, the dynamical networks (11) with time-varying delay satisfying (6) are exponentially stable if there exist symmetric positive definite matrices $P_{i}>0, Q_{i}>0, R_{i}>0, S_{i}>0, U_{i}>0, T_{i}>0, W_{i}>0$, and a matrix $L_{i}$ appropriately dimensioned such that the following symmetric linear matrix inequality holds:

$$
\begin{aligned}
& \Sigma_{i 1}=\Sigma_{i}-\left[\begin{array}{lllll}
0 & 0 & I & -I & 0
\end{array}\right]^{T} e^{-2 \alpha h_{2}} U_{i}\left[\begin{array}{lllll}
0 & 0 & I & -I & 0
\end{array}\right]<0, \\
& \Sigma_{i 2}=\Sigma_{i}-\left[\begin{array}{lllll}
0 & 0 & 0 & I & -I
\end{array}\right]^{T} e^{-2 \alpha h_{2}} U_{i}\left[\begin{array}{lllll}
0 & 0 & 0 & I & -I
\end{array}\right]<0 \text {, } \\
& \Sigma_{i 3}=\left[\begin{array}{cccc}
-0.5\left(e^{-2 \alpha h_{1}}+e^{-2 \alpha h_{2}}\right) R_{i} & 2 k_{1} \tilde{C}_{i} P_{i} & k_{2} L_{i}^{T} & 2 L_{i}^{T} \\
* & -2 k_{1} e^{-2 \alpha k_{1}} S_{i} & 0 & 0 \\
* & * & -k_{2} W_{i} & 0 \\
* & * & * & -2 e^{-2 \alpha d} T_{i}
\end{array}\right]<0 \text {, } \\
& \Sigma_{i 4}=\left[\begin{array}{ccccc}
-0.5 P_{i} & 2 k_{1} \tilde{C}_{i} P_{i} & d^{2} L_{i}^{T} & 3 D_{2 i}^{T} & 2 k_{2} D_{3 i}^{T} \\
* & -2 k_{1} e^{-2 \alpha k_{1}} S_{i} & 0 & 0 & 0 \\
* & * & -d^{2} T_{i} & 0 & 0 \\
* & * & * & -3 e^{-2 \alpha d} T_{i} & 0 \\
* & * & * & * & -2 k_{2} e^{-2 \alpha k_{2}} W_{i}
\end{array}\right]<0 \text {, }
\end{aligned}
$$

$i=2, \ldots, N$, where

$$
\begin{aligned}
\Sigma_{i}= & {\left[\begin{array}{ccccc}
\Sigma_{i 11} & \Sigma_{i 12} & \Sigma_{i 13} & \Sigma_{i 14} & \Sigma_{i 15} \\
* & \Sigma_{i 22} & 0 & \Sigma_{i 24} & 0 \\
* & * & \Sigma_{i 33} & \Sigma_{i 34} & 0 \\
* & * & * & \Sigma_{i 44} & \Sigma_{i 45} \\
* & * & * & * & \Sigma_{i 55}
\end{array}\right] } \\
\Sigma_{i 11}= & P_{i}^{T}\left(\tilde{A}_{i}+\alpha I\right)+\left(\tilde{A}_{i}+\alpha I\right)^{T} P_{i}-D_{1 i} L_{i}-L_{i}^{T} D_{1 i}^{T}+3 e^{2 \alpha d} D_{2 i}^{T} T_{i} D_{2 i} \\
& +2 k_{2} e^{2 \alpha k_{2}} D_{3 i}^{T} W_{i} D_{3 i}+2 Q_{i}+k_{1} S_{i}-0.5 e^{-2 \alpha h_{1}} R_{i}-0.5 e^{-2 \alpha h_{2}} R_{i}, \\
\Sigma_{i 12}= & P_{i} \tilde{A}_{i}^{T}, \\
\Sigma_{i 13}= & e^{-2 \alpha h_{1}} R_{i}, \\
\Sigma_{i 14}= & \tilde{B}_{i} P_{i} \\
\Sigma_{i 15}= & e^{-2 \alpha h_{2}} R_{i}, \\
\Sigma_{i 22}= & h_{1}^{2} R_{i}+h_{2}^{2} R_{i}+\eta^{2} U_{i}-1.5 P_{i},
\end{aligned}
$$




$$
\begin{aligned}
& \Sigma_{i 24}=\tilde{B}_{i} P_{i}, \\
& \Sigma_{i 33}=-e^{-2 \alpha h_{1}} Q_{i}-e^{-2 \alpha h_{1}} R_{i}-e^{-2 \alpha h_{2}} U_{i}, \\
& \Sigma_{i 34}=e^{-2 \alpha h_{2}} U_{i}, \\
& \Sigma_{i 44}=-2 e^{-2 \alpha h_{2}} U_{i}, \\
& \Sigma_{i 45}=e^{-2 \alpha h_{2}} U_{i}, \\
& \Sigma_{i 55}=-2 e^{-2 \alpha h_{2}} U_{i}-2 e^{-2 \alpha h_{2}} Q_{i}-2 e^{-2 \alpha h_{2}} R_{i},
\end{aligned}
$$

then the dynamical networks (11) have exponential synchronization. Moreover, the feedback control is

$$
u_{i}(t)=-L_{i} P_{i}^{-1} z_{i}(t)
$$

Proof Let $Y_{i}=P_{i}^{-1}, y_{i}(t)=Y_{i} z_{i}(t)$. Using the feedback control (16) we consider the following Lyapunov-Krasovskii functional:

$$
\begin{aligned}
V_{i}\left(z_{i}(t)\right)= & V_{i 1}(t)+V_{i 2}(t)+V_{i 3}(t)+V_{i 4}(t)+V_{i 5}(t)+V_{i 6}(t)+V_{i 7}(t) \\
& +V_{i 8}(t)+V_{i 9}(t)
\end{aligned}
$$

where

$$
\begin{aligned}
& V_{i 1}(t)=z_{i}^{T}(t) Y_{i} z_{i}(t), \\
& V_{i 2}(t)=\int_{t-h_{1}}^{t} e^{2 \alpha(s-t)} z_{i}^{T}(s) Y_{i} Q_{i} Y_{i} z_{i}(s) d s, \\
& V_{i 3}(t)=\int_{t-h_{2}}^{t} e^{2 \alpha(s-t)} z_{i}^{T}(s) Y_{i} Q_{i} Y_{i} z_{i}(s) d s, \\
& V_{i 4}(t)=h_{1} \int_{-h_{1}}^{0} \int_{t+s}^{t} e^{2 \alpha(\tau-t)} \dot{z}_{i}^{T}(\tau) Y_{i} R_{i} Y_{i} \dot{z}_{i}(\tau) d \tau d s, \\
& V_{i 5}(t)=h_{2} \int_{-h_{2}}^{0} \int_{t+s}^{t} e^{2 \alpha(\tau-t)} \dot{z}_{i}^{T}(\tau) Y_{i} R_{i} Y_{i} \dot{z}_{i}(\tau) d \tau d s, \\
& V_{i 6}(t)=\left(h_{2}-h_{1}\right) \int_{t-h_{2}}^{t-h_{1}} \int_{t+s}^{t} e^{2 \alpha(\tau-t)} \dot{z}_{i}^{T}(\tau) Y_{i} U_{i} Y_{i} \dot{z}_{i}(\tau) d \tau d s, \\
& V_{i 7}(t)=\int_{-k_{1}}^{0} \int_{t+s}^{t} e^{2 \alpha(\tau-t)} z_{i}^{T}(\tau) Y_{i} S_{i} Y_{i}(\tau) d \tau d s, \\
& V_{i 8}(t)=d \int_{-d}^{0} \int_{t+s}^{t} e^{2 \alpha(\tau-t)} \dot{z}_{i}^{T}(\tau) K_{i}^{T} T_{i}^{-1} K_{i} \dot{z}_{i}(\tau) d \tau d s, \\
& V_{i 9}(t)=\int_{-k_{2}}^{0} \int_{t+s}^{t} e^{2 \alpha(\tau-t)} z_{i}^{T}(\tau) K_{i}^{T} W_{i}^{-1} K_{i} z_{i}(\tau) d \tau d s .
\end{aligned}
$$

It easy to check that

$$
\gamma\left\|z_{i}(t)\right\|^{2} \leq V_{i}\left(z_{i}(t)\right), \quad \forall t \geq 0 .
$$


By taking the derivative of $V_{i 1}(t)$ along the trajectories of system (11), we have the following:

$$
\begin{aligned}
\dot{V}_{i 1}(t)= & 2 z_{i}^{T}(t) Y_{i} \dot{z}_{i}(t) \\
= & 2 y_{i}^{T}(t)\left[\left(\tilde{A}_{i}+D_{1 i} K_{i}\right) z_{i}(t)+\tilde{B}_{i} z_{i}(t-h(t))+\tilde{C}_{i} \int_{t-k_{1}(t)}^{t} z_{i}(s) d s\right. \\
& \left.+D_{2 i} K_{i} z_{i}(t-d(t))+D_{3 i} K_{i} \int_{t-k_{2}(t)}^{t} z_{i}(s) d s\right] \\
= & y_{i}^{T}(t)\left[P_{i} \tilde{A}_{i}+\tilde{A}_{i}^{T} P_{i}\right] y_{i}(t)+2 y_{i}^{T}(t) \tilde{B}_{i} P_{i} y_{i}(t-h(t)) \\
& +2 y_{i}^{T}(t) \tilde{C}_{i} P_{i} \int_{t-k_{1}(t)}^{t} y_{i}(s) d s-2 y_{i}^{T}(t) D_{i} L_{i}^{T} y_{i}(t)+2 y_{i}^{T}(t) D_{2 i} u_{i}(t-d(t)) \\
& +2 y_{i}^{T}(t) D_{3 i} \int_{t-k_{2}(t)}^{t} u_{i}(s) d s+2 y_{i}^{T}(t) \alpha P_{i} y_{i}(t)-2 y_{i}^{T}(t) \alpha P_{i} y_{i}(t) .
\end{aligned}
$$

Applying Lemma 2.4 and Lemma 2.3 gives

$$
\begin{aligned}
2 y_{i}^{T}(t) \tilde{C}_{i} P_{i} \int_{t-k_{1}(t)}^{t} y_{i}(s) d s \leq & 2 k_{1} e^{2 \alpha k_{1}} y_{i}^{T}(t) \tilde{C}_{i} P_{i} S_{i}^{-1} P_{i} \tilde{C}_{i}^{T} y_{i}(t) \\
& +\frac{e^{-2 \alpha k_{1}}}{2 k_{1}}\left(\int_{t-k_{1}(t)}^{t} y_{i}(s) d s\right)^{T} S_{i}\left(\int_{t-k_{1}(t)}^{t} y_{i}(s) d s\right) \\
\leq & 2 k_{1} e^{2 \alpha k_{1}} y_{i}^{T}(t) \tilde{C}_{i} P_{i} S_{i}^{-1} P_{i} \tilde{C}_{i}^{T} y_{i}(t) \\
& +\frac{1}{2} e^{-2 \alpha k_{1}} \int_{t-k_{1}(t)}^{t} y_{i}^{T}(s) S_{i} y_{i}(s) d s, \\
2 y_{i}^{T}(t) D_{2 i} u_{i}(t-d(t)) \leq 3 & e^{2 \alpha d} y_{i}^{T}(t) D_{2 i} T_{i} D_{2 i}^{T} y_{i}(t) \\
+ & \frac{e^{-2 \alpha d}}{3} u_{i}^{T}(t-d(t)) T_{i}^{-1} u_{i}(t-d(t)), \\
2 y_{i}^{T}(t) D_{3 i} \int_{t-k_{2}(t)}^{t} u_{i}(s) d s \leq & 2 k_{2} e^{2 \alpha k_{2}} y_{i}^{T}(t) D_{3 i} W_{i} D_{3 i}^{T} y_{i}(t) \\
& +\frac{e^{2 \alpha k_{2}}}{2 k_{2}}\left(\int_{t-k_{2}(t)}^{t} u_{i}(s) d s\right)^{T} W_{i}^{-1}\left(\int_{t-k_{2}(t)}^{t} u_{i}(s) d s\right) \\
\leq & 2 k_{2} e^{2 \alpha k_{2}} y_{i}^{T}(t) D_{3 i} W_{i} D_{3 i}^{T} y_{i}(t) \\
& +\frac{e^{2 \alpha k_{2}}}{2} \int_{t-k_{2}(t)}^{t} u_{i}^{T}(s) W_{i}^{-1} u_{i}(s) d s .
\end{aligned}
$$

Therefore

$$
\begin{aligned}
\dot{V}_{i 1}(t)+2 \alpha V_{i 1}(t) \leq & y_{i}^{T}(t)\left[P_{i} \tilde{A}_{i}+\tilde{A}_{i}^{T} P_{i}\right] y_{i}(t)+2 y_{i}^{T}(t) \alpha P_{i} y_{i}(t) \\
& +2 y_{i}^{T}(t) \tilde{B}_{i} P_{i} y_{i}(t-h(t))-2 y_{i}^{T}(t) D_{i} L_{i}^{T} y_{i}(t) \\
& +2 k_{1} e^{2 \alpha k_{1}} y_{i}^{T}(t) \tilde{C}_{i} P_{i} S_{i}^{-1} P_{i} \tilde{C}_{i}^{T} y_{i}(t) \\
& +\frac{1}{2} e^{-2 \alpha k_{1}} \int_{t-k_{1}(t)}^{t} y_{i}^{T}(s) S_{i} y_{i}(s) d s \\
& +3 e^{2 \alpha d} y_{i}^{T}(t) D_{2 i} T_{i} D_{2 i}^{T} y_{i}(t) \\
& +\frac{e^{-2 \alpha d}}{3} u_{i}^{T}(t-d(t)) T_{i}^{-1} u_{i}(t-d(t))
\end{aligned}
$$




$$
\begin{aligned}
& +2 k_{2} e^{2 \alpha k_{2}} y_{i}^{T}(t) D_{3 i} W_{i} D_{3 i}^{T} y_{i}(t) \\
& +\frac{e^{2 \alpha k_{2}}}{2} \int_{t-k_{2}(t)}^{t} u_{i}^{T}(s) W_{i}^{-1} u_{i}(s) d s .
\end{aligned}
$$

Next, by taking the derivative of $V_{i j}(t), j=2,3, \ldots, 9$ along the trajectories of system (11), we have the following:

$$
\begin{aligned}
\dot{V}_{i 2}(t) & \leq y_{i}^{T}(t) Q_{i} y_{i}(t)-e^{-2 \alpha h_{1}} y_{i}^{T}\left(t-h_{1}\right) Q_{i} y_{i}\left(t-h_{1}\right)-2 \alpha V_{i 2}(t), \\
\dot{V}_{i 3}(t) & \leq y_{i}^{T}(t) Q_{i} y_{i}(t)-e^{-2 \alpha h_{2}} y_{i}^{T}\left(t-h_{2}\right) Q_{i} y_{i}\left(t-h_{2}\right)-2 \alpha V_{i 3}(t), \\
\dot{V}_{i 4}(t) & \leq h_{1}^{2} \dot{y}_{i}^{T}(t) R_{i} \dot{y}_{i}(t)-h_{1} e^{-2 \alpha h_{1}} \int_{t-h_{1}}^{t} \dot{y}_{i}^{T}(s) R_{i} \dot{y}_{i}(s) d s-2 \alpha V_{i 4}(t), \\
\dot{V}_{i 5}(t) & \leq h_{2}^{2} \dot{y}_{i}^{T}(t) R_{i} \dot{y}_{i}(t)-h_{2} e^{-2 \alpha h_{2}} \int_{t-h_{2}}^{t} \dot{y}_{i}^{T}(s) R_{i} \dot{y}_{i}(s) d s-2 \alpha V_{i 5}(t), \\
\dot{V}_{i 6}(t) & \leq \eta^{2} \dot{y}_{i}^{T}(t) U_{i} \dot{y}_{i}(t)-\eta e^{-2 \alpha h_{2}} \int_{t-h_{2}}^{t-h_{1}} \dot{y}_{i}^{T}(s) U_{i} \dot{y}_{i}(s) d s-2 \alpha V_{i 6}(t), \\
\dot{V}_{i 7}(t) & \leq k_{1} y_{i}^{T}(t) S_{i} y_{i}(t)-e^{-2 \alpha k_{1}} \int_{t-k_{1}(t)}^{t} y_{i}^{T}(s) S_{i} y_{i}(s) d s-2 \alpha V_{i 7}(t), \\
\dot{V}_{i 8}(t) & \leq d^{2} \dot{z}_{i}^{T}(t) K_{i}^{T} T_{i}^{-1} K_{i} \dot{z}_{i}^{T}(t)-d e^{-2 \alpha d} \int_{t-d}^{t} \dot{z}_{i}^{T}(s) K_{i}^{T} T_{i}^{-1} K_{i} \dot{z}_{i}^{T}(s) d s-2 \alpha V_{i 8}(t) \\
& \leq k_{2} y_{i}^{T}(t) L_{i}^{T} W_{i}^{-1} L_{i} y_{i}^{T}(t)-e^{-2 \alpha k_{2}} \int_{t-k_{2}(t)}^{t} u_{i}^{T}(s) W_{i}^{-1} u_{i}^{T}(s) d s-2 \alpha V_{i 9}(t) . \\
& \leq d^{2} \dot{y}_{i}^{T}(t) P_{i} K_{i}^{T} T_{i}^{-1} K_{i} P_{i} \dot{y}_{i}^{T}(t)-d(t) e^{-2 \alpha d} \int_{t-d(t)}^{t} \dot{u}_{i}^{T}(s) T_{i}^{-1} \dot{u}_{i}(s) d s-2 \alpha V_{i 8}(t) \\
\dot{V}_{i 9}(t) & \leq k_{2} z_{i}^{T}(t) K_{i}^{T} W_{i}^{-1} K_{i} z_{i}^{T}(t)-e^{-2 \alpha k_{2}} \int_{t-k_{2}}^{t} z_{i}^{T}(s) K_{i}^{T} W_{i}^{-1} K_{i} z_{i}^{T}(s) d s-2 \alpha V_{i 9}(t) \\
& =d^{2} \dot{y}_{i}^{T}(t) L_{i}^{T} T_{i}^{-1} L_{i} \dot{y}_{i}^{T}(t)-d(t) e_{i}^{-2 \alpha d} \int_{i}^{t} \dot{u}_{i}^{T}(s) T_{i}^{-1} \dot{u}_{i}(s) d s-2 \alpha V_{i 8}(t), \\
& \\
&
\end{aligned}
$$

Applying Lemma 2.3 and the Leibniz-Newton formula, we have

$$
\begin{aligned}
-h_{1} \int_{t-h_{1}}^{t} \dot{y}_{i}^{T}(s) R_{i} \dot{y}_{i}(s) d s \leq & -\left[\int_{t-h_{1}}^{t} \dot{y}_{i}(s) d s\right]^{T} R_{i}\left[\int_{t-h_{1}}^{t} \dot{y}_{i}(s) d s\right] \\
\leq & -\left[y_{i}(t)-y_{i}\left(t-h_{1}\right)\right]^{T} R_{i}\left[y_{i}(t)-y_{i}\left(t-h_{1}\right)\right] \\
= & -y_{i}^{T}(t) R_{i} y_{i}(t)+2 y_{i}^{T}(t) R_{i} y_{i}\left(t-h_{1}\right) \\
& -y_{i}^{T}\left(t-h_{1}\right) R_{i} y_{i}\left(t-h_{1}\right)
\end{aligned}
$$

and

$$
\begin{aligned}
-h_{2} \int_{t-h_{2}}^{t} \dot{y}_{i}^{T}(s) R_{i} \dot{y}_{i}(s) d s & \leq-\left[\int_{t-h_{2}}^{t} \dot{y}_{i}(s) d s\right]^{T} R_{i}\left[\int_{t-h_{2}}^{t} \dot{y}_{i}(s) d s\right] \\
& \leq-\left[y_{i}(t)-y_{i}\left(t-h_{2}\right)\right]^{T} R_{i}\left[y_{i}(t)-y_{i}\left(t-h_{2}\right)\right]
\end{aligned}
$$




$$
\begin{aligned}
= & -y_{i}^{T}(t) R_{i} y_{i}(t)+2 y_{i}^{T}(t) R_{i} y_{i}\left(t-h_{2}\right) \\
& -y_{i}^{T}\left(t-h_{2}\right) R_{i} y_{i}\left(t-h_{2}\right) .
\end{aligned}
$$

On the other hand,

$$
\begin{aligned}
-\left(h_{2}-h_{1}\right) \int_{t-h_{2}}^{t-h_{1}} \dot{y}_{i}^{T}(s) U_{i} \dot{y}_{i}(s) d s= & -\left(h_{2}-h_{1}\right) \int_{t-h_{2}}^{t-h(t)} \dot{y}_{i}^{T}(s) U_{i} \dot{y}_{i}(s) d s \\
& -\left(h_{2}-h_{1}\right) \int_{t-h(t)}^{t-h_{1}} \dot{y}_{i}^{T}(s) U_{i} \dot{y}_{i}(s) d s \\
= & -\left(h_{2}-h(t)\right) \int_{t-h_{2}}^{t-h(t)} \dot{y}_{i}^{T}(s) U_{i} \dot{y}_{i}(s) d s \\
& -\left(h(t)-h_{1}\right) \int_{t-h_{2}}^{t-h(t)} \dot{y}_{i}^{T}(s) U_{i} \dot{y}_{i}(s) d s \\
& -\left(h(t)-h_{1}\right) \int_{t-h(t)}^{t-h_{1}} \dot{y}_{i}^{T}(s) U_{i} \dot{y}_{i}(s) d s \\
& -\left(h_{2}-h(t)\right) \int_{t-h(t)}^{t-h_{1}} \dot{y}_{i}^{T}(s) U_{i} \dot{y}_{i}(s) d s .
\end{aligned}
$$

Using Lemma 2.3 gives

$$
\begin{aligned}
-\left(h_{2}-h(t)\right) \int_{t-h_{2}}^{t-h(t)} \dot{y}_{i}^{T}(s) U_{i} \dot{y}_{i}(s) d s \leq & -\left[\int_{t-h_{2}}^{t-h(t)} \dot{y}_{i}(s) d s\right]^{T} U_{i}\left[\int_{t-h_{2}}^{t-h(t)} \dot{y}_{i}(s) d s\right] \\
\leq & -\left[y_{i}(t-h(t))-y_{i}\left(t-h_{2}\right)\right]^{T} U_{i} \\
& \times\left[y_{i}(t-h(t))-y_{i}\left(t-h_{2}\right)\right] \\
= & -y_{i}^{T}(t-h(t)) U_{i} y_{i}(t-h(t)) \\
& +2 y_{i}^{T}(t-h(t)) U_{i} y_{i}\left(t-h_{2}\right) \\
& -y_{i}^{T}\left(t-h_{2}\right) U_{i} y_{i}\left(t-h_{2}\right)
\end{aligned}
$$

and

$$
\begin{aligned}
-\left(h(t)-h_{1}\right) \int_{t-h(t)}^{t-h_{1}} \dot{y}_{i}^{T}(s) U_{i} \dot{y}_{i}(s) d s \leq & -\left[\int_{t-h(t)}^{t-h_{1}} \dot{y}_{i}(s) d s\right]^{T} U_{i}\left[\int_{t-h(t)}^{t-h_{1}} \dot{y}_{i}(s) d s\right] \\
\leq & -\left[y_{i}\left(t-h_{1}\right)-y_{i}(t-h(t))\right]^{T} U_{i} \\
& \times\left[y_{i}\left(t-h_{1}\right)-y_{i}(t-h(t))\right] \\
= & -y_{i}^{T}\left(t-h_{1}\right) U_{i} y_{i}\left(t-h_{1}\right)+2 y_{i}^{T}\left(t-h_{1}\right) U_{i} y_{i}(t-h(t)) \\
& -y_{i}^{T}(t-h(t)) U_{i} y_{i}(t-h(t)) .
\end{aligned}
$$

Let $\beta=\frac{h_{2}-h(t)}{h_{2}-h_{1}} \leq 1$. Then

$$
\begin{aligned}
-\left(h_{2}-h(t)\right) \int_{t-h(t)}^{t-h_{1}} \dot{y}_{i}^{T}(s) U_{i} \dot{y}_{i}(s) d s & =-\beta \int_{t-h(t)}^{t-h_{1}}\left(h_{2}-h_{1}\right) \dot{y}_{i}^{T}(s) U_{i} \dot{y}_{i}(s) d s \\
& \leq-\beta \int_{t-h(t)}^{t-h_{1}}\left(h(t)-h_{1}\right) \dot{y}_{i}^{T}(s) U_{i} \dot{y}_{i}(s) d s
\end{aligned}
$$




$$
\begin{aligned}
\leq & -\beta\left[y_{i}\left(t-h_{1}\right)-y_{i}(t-h(t))\right]^{T} U_{i} \\
& \times\left[y_{i}\left(t-h_{1}\right)-y_{i}(t-h(t))\right]
\end{aligned}
$$

and

$$
\begin{aligned}
-\left(h(t)-h_{1}\right) \int_{t-h_{2}}^{t-h(t)} \dot{y}_{i}^{T}(s) U_{i} \dot{y}_{i}(s) d s= & -(1-\beta) \int_{t-h_{2}}^{t-h(t)}\left(h_{2}-h_{1}\right) \dot{y}_{i}^{T}(s) U_{i} \dot{y}_{i}(s) d s \\
\leq & -(1-\beta) \int_{t-h_{2}}^{t-h(t)}\left(h_{2}-h(t)\right) \dot{y}_{i}^{T}(s) U_{i} \dot{y}_{i}(s) d s \\
\leq & -(1-\beta)\left[y_{i}(t-h(t))-y_{i}\left(t-h_{2}\right)\right]^{T} U_{i} \\
& \times\left[y_{i}(t-h(t))-y_{i}\left(t-h_{2}\right)\right] .
\end{aligned}
$$

Therefore from (23)-(26), we obtain

$$
\begin{aligned}
-\left(h_{2}-h_{1}\right) \int_{t-h_{2}}^{t-h_{1}} \dot{y}_{i}^{T}(s) U_{i} \dot{y}_{i}(s) d s \leq & -\left[y_{i}(t-h(t))-y_{i}\left(t-h_{2}\right)\right]^{T} U_{i} \\
& \times\left[y_{i}(t-h(t))-y_{i}\left(t-h_{2}\right)\right] \\
& -\left[y_{i}\left(t-h_{1}\right)-y_{i}(t-h(t))\right]^{T} U_{i} \\
& \times\left[y_{i}\left(t-h_{1}\right)-y_{i}(t-h(t))\right] \\
& -\beta\left[y_{i}\left(t-h_{1}\right)-y_{i}(t-h(t))\right]^{T} U_{i} \\
& \times\left[y_{i}\left(t-h_{1}\right)-y_{i}(t-h(t))\right] \\
& -(1-\beta)\left[y_{i}(t-h(t))-y_{i}\left(t-h_{2}\right)\right]^{T} U_{i} \\
& \times\left[y_{i}(t-h(t))-y_{i}\left(t-h_{2}\right)\right] .
\end{aligned}
$$

From $\dot{V}_{i 8}(t)$, applying Lemma 2.3 and the Leibniz-Newton formula gives

$$
\begin{aligned}
-d(t) e^{-2 \alpha d} \int_{t-d(t)}^{t} \dot{u}_{i}^{T}(s) T_{i}^{-1} \dot{u}_{i}(s) d s \leq & -e^{-2 \alpha d}\left(\int_{t-d(t)}^{t} \dot{u}_{i}(s) d s\right)^{T} T_{i}^{-1}\left(\int_{t-d(t)}^{t} \dot{u}_{i}(s) d s\right) \\
\leq & -e^{-2 \alpha d} u_{i}^{T}(t) T_{i}^{-1} u_{i}(t) \\
& +2 e^{-2 \alpha d} u_{i}^{T}(t) T_{i}^{-1} u_{i}(t-d(t)) \\
& -e^{-2 \alpha d} u_{i}^{T}(t-d(t)) T_{i}^{-1} u_{i}(t-d(t)) \\
\leq & -e^{-2 \alpha d} u_{i}^{T}(t) T_{i}^{-1} u_{i}(t)+3 e^{-2 \alpha d} u_{i}^{T}(t) T_{i}^{-1} u_{i}(t) \\
& +\frac{e^{-2 \alpha d}}{3} u_{i}^{T}(t-d(t)) T_{i}^{-1} T_{i} T_{i}^{-1} u_{i}(t-d(t)) \\
& -e^{-2 \alpha d} u_{i}^{T}(t-d(t)) T_{i}^{-1} u_{i}(t-d(t)) \\
= & 2 e^{-2 \alpha d} z_{i}^{T}(t) K_{i}^{T} T_{i}^{-1} K_{i} z_{i}(t) \\
& +\frac{e^{-2 \alpha d}}{3} u_{i}^{T}(t-d(t)) T_{i}^{-1} T_{i} T_{i}^{-1} u_{i}(t-d(t)) \\
& -e^{-2 \alpha d} u_{i}^{T}(t-d(t)) T_{i}^{-1} u_{i}(t-d(t))
\end{aligned}
$$




$$
\begin{aligned}
= & 2 e^{-2 \alpha d} y_{i}^{T}(t) L_{i}^{T} T_{i}^{-1} L_{i} y_{i}(t) \\
& +\frac{e^{-2 \alpha d}}{3} u_{i}^{T}(t-d(t)) T_{i}^{-1} u_{i}(t-d(t)) \\
& -e^{-2 \alpha d} u_{i}^{T}(t-d(t)) T_{i}^{-1} u_{i}(t-d(t)) .
\end{aligned}
$$

By using the following identity relation:

$$
\begin{aligned}
& -\dot{z}_{i}(t)+\left(\tilde{A}_{i}+D_{1 i} K_{i}\right) z_{i}(t)+\tilde{B}_{i} z_{i}(t-h(t))+\tilde{C}_{i} \int_{t-k_{1}(t)}^{t} z_{i}(s) d s \\
& +D_{2 i} K_{i} z_{i}(t-d(t))+D_{3 i} K_{i} \int_{t-k_{2}(t)}^{t} z_{i}(s) d s=0,
\end{aligned}
$$

we have

$$
\begin{aligned}
& -2 \dot{y}_{i}^{T}(t) P_{i} \dot{y}_{i}(t)+2 \dot{y}_{i}^{T}(t) \tilde{A}_{i} P_{i} y_{i}(t)-2 \dot{y}_{i}^{T}(t) D_{1 i} L_{i} y_{i}(t)+2 \dot{y}_{i}^{T}(t) \tilde{B}_{i} P_{i} y_{i}(t-h(t)) \\
& \quad+2 \dot{y}_{i}^{T}(t) \tilde{C}_{i} P_{i} \int_{t-k_{1}(t)}^{t} y_{i}(s) d s+2 \dot{y}_{i}^{T}(t) D_{2 i} u_{i}(t-d(t)) \\
& \quad+2 \dot{y}_{i}^{T}(t) D_{3 i} \int_{t-k_{2}(t)}^{t} u_{i}(s) d s=0 .
\end{aligned}
$$

Applying Lemma 2.4 and Lemma 2.3 gives

$$
\begin{aligned}
& 2 \dot{y}_{i}^{T}(t) \tilde{C}_{i} P_{i} \int_{t-k_{1}(t)}^{t} y_{i}(s) d s \leq 2 k_{1} e^{2 \alpha k_{1}} \dot{y}_{i}^{T}(t) \tilde{C}_{i} P_{i} S_{i}^{-1} P_{i} \tilde{C}_{i}^{T} \dot{y}_{i}(t) \\
&+\frac{1}{2 k_{1}} e^{-2 \alpha k_{1}}\left(\int_{t-k_{1}(t)}^{t} y_{i}(s) d s\right)^{T} S_{i} \\
& \times\left(\int_{t-k_{1}(t)}^{t} y_{i}(s) d s\right) \\
& \leq 2 k_{1} e^{2 \alpha k_{1}} \dot{y}_{i}^{T}(t) \tilde{C}_{i} P_{i} S_{i}^{-1} P_{i} \tilde{C}_{i}^{T} \dot{y}_{i}(t) \\
&+\frac{1}{2} e^{-2 \alpha k_{1}} \int_{t-k_{1}(t)}^{t} y_{i}^{T}(s) S_{i} y_{i}(s) d s, \\
& 2 \dot{y}_{i}^{T}(t) D_{2 i} u_{i}(t-d(t)) \leq 3 e^{2 \alpha d} \dot{y}_{i}^{T}(t) D_{2 i}^{T} T_{i}^{-1} D_{2 i} \dot{y}_{i}(t) \\
&+\frac{e^{-2 \alpha d}}{3} u_{i}^{T}(t-d(t)) T_{i}^{-1} u_{i}(t-d(t)), \\
& 3 \dot{y}_{i}^{T}(t) D_{3 i} \int_{t-k_{2}(t)}^{t} u_{i}(s) d s \leq 2 k_{2} e^{2 \alpha k_{2}} y_{i}^{T}(t) D_{3 i}^{T} W_{i}^{-1} D_{3 i} y_{i}(t) \\
&+\frac{1}{2 k_{2}} e^{-2 \alpha k_{2}}\left(\int_{t-k_{2}(t)}^{t} u_{i}(s) d s\right)^{T} W_{i} \\
& \times\left(\int_{t-k_{2}(t)}^{t} u_{i}(s) d s\right) \\
& \leq 2 k_{2} e^{2 \alpha k_{2}} y_{i}^{T}(t) D_{3 i}^{T} W_{i}^{-1} D_{3 i} y_{i}(t) \\
&+\frac{e^{2 \alpha k_{2}}}{2} \int_{t-k_{2}(t)}^{t} u_{i}^{T}(s) W_{i}^{-1} u_{i}(s) d s .
\end{aligned}
$$


Hence, according to (19)-(28), (30)-(32), and adding the zero items of (29) we have

$$
\begin{aligned}
\dot{V}_{i}\left(z_{i}(t)\right)+2 \alpha V_{i}\left(z_{i}(t)\right) \leq & \xi_{i}^{T}(t)\left[(1-\beta) \Sigma_{1 i}+\beta \Sigma_{2 i}\right] \xi_{i}(t)+y_{i}^{T}(t) \mathcal{M}_{3 i} y_{i}(t) \\
& +\dot{y}_{i}^{T}(t) \mathcal{M}_{4 i} \dot{y}_{i}(t)
\end{aligned}
$$

where $\Sigma_{1 i}$ and $\Sigma_{2 i}$ are defined as in (12) and (13), respectively, and

$$
\begin{aligned}
& \xi_{i}^{T}(t)=\left[\begin{array}{lllll}
y_{i}^{T}(t) & \dot{y}_{i}^{T}(t) & y_{i}^{T}\left(t-h_{1}\right) & y_{i}^{T}(t-h(t)) & y_{i}^{T}\left(t-h_{2}\right)
\end{array}\right], \\
& \mathcal{M}_{3 i}=-0.5\left(e^{-2 \alpha h_{1}}+e^{-2 \alpha h_{2}}\right) R_{i}+2 k_{1} e^{2 \alpha k_{1}} \tilde{C}_{i} P_{i} S_{i}^{-1} P_{i} \tilde{C}_{i}^{T}+k_{2} L_{i}^{T} W_{i}^{-1} L_{i} \\
& +2 e^{-2 \alpha d} L_{i}^{T} T_{i}^{-1} L_{i} \\
& \mathcal{M}_{4 i}=-0.5 P_{i}+2 k_{1} e^{2 \alpha k_{1}} \tilde{C}_{i} P_{i} S_{i}^{-1} P_{i} \tilde{C}_{i}^{T}+d^{2} L_{i}^{T} T_{i}^{-1} L_{i}+3 e^{2 \alpha d} D_{2 i}^{T} T_{i}^{-1} D_{2 i} \\
& +2 k_{2} e^{2 \alpha k_{2}} D_{3 i}^{T} W_{i}^{-1} D_{3 i} \text {. }
\end{aligned}
$$

By $(1-\beta) \Sigma_{1 i}+\beta \Sigma_{2 i}<0$ holds if and only if $\Sigma_{1 i}<0$ and $\Sigma_{2 i}<0$. Applying the Schur complement lemma, the inequalities $\mathcal{M}_{3 i}<0$ and $\mathcal{M}_{4 i}<0$ are equivalent to $\Sigma_{3 i}<0$ and $\Sigma_{4 i}<0$, respectively. Therefore, it follows from (12)-(15), and (33), we obtain

$$
\dot{V}_{i}\left(z_{i}(t)\right)+2 \alpha V_{i}\left(z_{i}(t)\right) \leq 0, \quad \forall t \geq 0
$$

Integrating both sides of (34) from 0 to $t$, we have

$$
V_{i}\left(z_{i}(t)\right) \leq V_{i}\left(z_{i}(0)\right) e^{-2 \alpha t}, \quad \forall t \geq 0
$$

On the other hand, using the condition (18), we have

$$
\left\|z_{i}(t)\right\| \leq \sqrt{\frac{V_{i}\left(z_{i}(0)\right)}{\gamma}} e^{-\alpha t}, \quad \forall t \geq 0
$$

Estimating $V_{i}\left(z_{i}(0)\right)$ gives

$$
\begin{aligned}
V_{i 1}\left(z_{i}(0)\right) & =z_{i}^{T}(0) P_{i}^{-1} z_{i}(0) \leq \lambda_{\max }\left(P_{i}^{-1}\right)\left\|\phi_{i}\right\|^{2}, \\
V_{i 2}\left(z_{i}(0)\right) & =\int_{-h_{1}}^{0} e^{2 \alpha s} z_{i}^{T}(s) Y_{i} Q_{i} Y_{i} z_{i}(s) d s \leq \lambda_{\max }\left(P_{i}^{-1} Q_{i} P_{i}^{-1}\right) \int_{-h_{1}}^{0} e^{2 \alpha s} d s\left\|\varphi_{i}\right\|^{2} \\
& =\lambda_{\max }\left(P_{i}^{-1} Q_{i} P_{i}^{-1}\right) \frac{1-e^{-2 \alpha h_{1}}}{2 \alpha}\left\|\varphi_{i}\right\|^{2} \leq \lambda_{\max }\left(P_{i}^{-1} Q_{i} P_{i}^{-1}\right) \frac{1-e^{-2 \alpha h_{2}}}{2 \alpha}\left\|\varphi_{i}\right\|^{2}, \\
V_{i 3}\left(z_{i}(0)\right) & \leq \lambda_{\max }\left(P_{i}^{-1} Q_{i} P_{i}^{-1}\right) \frac{1-e^{-2 \alpha h_{2}}}{2 \alpha}\left\|\varphi_{i}\right\|^{2}, \\
V_{i 4}\left(z_{i}(0)\right) & =h_{1} \int_{-h_{1}}^{0} \int_{s}^{0} e^{2 \alpha \tau} \dot{z}_{i}^{T}(\tau) Y_{i} R_{i} Y_{i} \dot{z}_{i}(\tau) d \tau d s \\
& =h_{1} \int_{-h_{1}}^{0} e^{2 \alpha s}\left[z_{i}^{T}(0) Y_{i} R_{i} Y_{i} z_{i}(0)-z_{i}^{T}(s) Y_{i} R_{i} Y_{i} z_{i}(s)\right] d s \\
& \leq h_{2} \lambda_{\max }\left(Y_{i} R_{i} Y_{i}\right) \int_{-h_{1}}^{0} e^{2 \alpha s} d s\left\|\phi_{i}\right\|^{2}-h_{2} \lambda_{\max }\left(Y_{i} R_{i} Y_{i}\right) \int_{-h_{1}}^{0} e^{2 \alpha s} d s\left\|\varphi_{i}\right\|^{2}
\end{aligned}
$$




$$
\begin{aligned}
& =h_{2} \lambda_{\max }\left(Y_{i} R_{i} Y_{i}\right) \frac{1-e^{-2 \alpha h_{1}}}{2 \alpha}\left\|\phi_{i}\right\|^{2}-h_{2} \lambda_{\max }\left(Y_{i} R_{i} Y_{i}\right) \frac{1-e^{-2 \alpha h_{1}}}{2 \alpha}\left\|\varphi_{i}\right\|^{2} \\
& \leq h_{2} \lambda_{\max }\left(P_{i}^{-1} R_{i} P_{i}^{-1}\right) \frac{1-e^{-2 \alpha h_{2}}}{2 \alpha}\left\|\phi_{i}\right\|^{2}+h_{2} \lambda_{\max }\left(P_{i}^{-1} R_{i} P_{i}^{-1}\right) \\
& \times \frac{1-e^{-2 \alpha h_{2}}}{2 \alpha}\left\|\varphi_{i}\right\|^{2} \\
& V_{i 5}\left(z_{i}(0)\right) \leq h_{2} \lambda_{\max }\left(P_{i}^{-1} R_{i} P_{i}^{-1}\right) \frac{1-e^{-2 \alpha h_{2}}}{2 \alpha}\left\|\phi_{i}\right\|^{2}+h_{2} \lambda_{\max }\left(P_{i}^{-1} R_{i} P_{i}^{-1}\right) \\
& \times \frac{1-e^{-2 \alpha h_{2}}}{2 \alpha}\left\|\varphi_{i}\right\|^{2} \\
& V_{i 6}\left(z_{i}(0)\right) \leq h_{2} \lambda_{\max }\left(P_{i}^{-1} U_{i} P_{i}^{-1}\right) \frac{1-e^{-2 \alpha h_{2}}}{2 \alpha}\left\|\phi_{i}\right\|^{2}+h_{2} \lambda_{\max }\left(P_{i}^{-1} U_{i} P_{i}^{-1}\right) \\
& \times \frac{1-e^{-2 \alpha h_{2}}}{2 \alpha}\left\|\varphi_{i}\right\|^{2} \\
& V_{i 7}\left(z_{i}(0)\right)=\int_{-k_{1}}^{0} \int_{s}^{0} e^{2 \alpha \tau} z_{i}^{T}(\tau) Y_{i} S_{i} Y_{i} z_{i}(\tau) d \tau d s \\
& \leq \int_{-k_{1}}^{0} \int_{-k_{1}}^{0} e^{2 \alpha s} z_{i}^{T}(\tau) Y_{i} S_{i} Y_{i} z_{i}(\tau) d \tau d s \\
& \leq \lambda_{\max }\left(Y_{i} S_{i} Y_{i}\right) \int_{-k_{1}}^{0} \int_{-k_{1}}^{0} e^{2 \alpha \tau} d \tau d s\left\|\varphi_{i}\right\|^{2} \\
& =k_{1} \lambda_{\max }\left(P_{i}^{-1} S_{i} P_{i}^{-1}\right) \frac{1-e^{-2 \alpha k_{1}}}{2 \alpha}\left\|\varphi_{i}\right\|^{2} \text {, } \\
& V_{i 8}\left(z_{i}(0)\right) \leq d \lambda_{\max }\left(P_{i}^{-1} L_{i}^{T} T_{i}^{-1} L_{i} P_{i}^{-1}\right) \frac{1-e^{-2 \alpha d}}{2 \alpha}\left\|\phi_{i}\right\|^{2} \\
& +d \lambda_{\max }\left(P_{i}^{-1} L_{i}^{T} T_{i}^{-1} L_{i} P_{i}^{-1}\right) \frac{1-e^{-2 \alpha d}}{2 \alpha}\left\|\varphi_{i}\right\|^{2}, \\
& V_{i 9}\left(z_{i}(0)\right) \leq k_{2} \lambda_{\max }\left(P_{i}^{-1} L_{i}^{T} W_{i}^{-1} L_{i} P_{i}^{-1}\right) \frac{1-e^{-2 \alpha k_{2}}}{2 \alpha}\left\|\varphi_{i}\right\|^{2},
\end{aligned}
$$

we have

$$
\left\|z_{i}(t)\right\| \leq \sqrt{\frac{\mathcal{N}}{\gamma}} e^{-\alpha t}, \quad \forall t \geq 0,
$$

which implies the dynamical networks (11) is globally exponentially stable under the controller H1, then exponential synchronization of the controlled dynamical networks (4) is achieved. The proof is thus completed.

\subsection{Intermittent delayed feedback control}

Theorem 3.4 For some given scalars $0<\alpha<\varepsilon$, the dynamical networks (7) with timevarying delay satisfying (6) are exponentially stable if there exist symmetric positive definite matrices $P_{i}>0, Q_{i}>0, R_{i}>0, S_{i}>0, U_{i}>0, T_{i}>0, W_{i}>0$, and a matrix $L_{i}$ with appropriately dimensioned such that the following symmetric linear matrix inequality holds:

$$
\begin{aligned}
& \Pi_{i 1}=\Pi_{i}-\left[\begin{array}{lllll}
0 & 0 & I & -I & 0
\end{array}\right]^{T} e^{-2 \alpha h_{2}} U_{i}\left[\begin{array}{lllll}
0 & 0 & I & -I & 0
\end{array}\right]<0 \\
& \Pi_{i 2}=\Pi_{i}-\left[\begin{array}{lllllll}
0 & 0 & 0 & I & -I
\end{array}\right]^{T} e^{-2 \alpha h_{2}} U_{i}\left[\begin{array}{lllll}
0 & 0 & 0 & I & -I
\end{array}\right]<0
\end{aligned}
$$




$$
\begin{aligned}
& \Pi_{i 3}=\tilde{\Pi}_{i}-\left[\begin{array}{lllll}
0 & 0 & I & -I & 0
\end{array}\right]^{T} e^{-2 \alpha h_{2}} U_{i}\left[\begin{array}{lllll}
0 & 0 & I & -I & 0
\end{array}\right]<0, \\
& \Pi_{i 4}=\tilde{\Pi}_{i}-\left[\begin{array}{lllll}
0 & 0 & 0 & I & -I
\end{array}\right]^{T} e^{-2 \alpha h_{2}} U_{i}\left[\begin{array}{lllll}
0 & 0 & 0 & I & -I
\end{array}\right]<0, \\
& \Pi_{i 5}=\left[\begin{array}{cccc}
-0.5\left(e^{-2 \alpha h_{1}}+e^{-2 \alpha h_{2}}\right) R_{i} & 2 k_{1} \tilde{C}_{i} P_{i} & k_{2} L_{i}^{T} & 2 L_{i}^{T} \\
* & -2 k_{1} e^{-2 \alpha k_{1}} S_{i} & 0 & 0 \\
* & * & -k_{2} W_{i} & 0 \\
* & * & * & -2 e^{-2 \alpha d} T_{i}
\end{array}\right]<0 \text {, } \\
& \Pi_{i 6}=\left[\begin{array}{ccccc}
-0.5 P_{i} & 2 k_{1} \tilde{C}_{i} P_{i} & d^{2} L_{i}^{T} & 3 D_{5 i}^{T} & 2 k_{2} D_{6 i}^{T} \\
* & -2 k_{1} e^{-2 \alpha k_{1}} S_{i} & 0 & 0 & 0 \\
* & * & -d^{2} T_{i} & 0 & 0 \\
* & * & * & -3 e^{-2 \alpha d} T_{i} & 0 \\
* & * & * & * & -2 k_{2} e^{-2 \alpha k_{2}} W_{i}
\end{array}\right]<0 \text {, } \\
& \Pi_{i 7}=\left[\begin{array}{cc}
-0.5\left(e^{-2 \alpha h_{1}}+e^{-2 \alpha h_{2}}\right) R_{i}-2 \varepsilon P_{i} & 2 k_{1} \tilde{C}_{i} P_{i} \\
* & -2 k_{1} e^{-2 \alpha k_{1}} S_{i}
\end{array}\right]<0 \text {, } \\
& \Pi_{i 8}=\left[\begin{array}{cc}
-0.5 P_{i} & 2 k_{1} \tilde{C}_{i} P_{i} \\
* & -2 k_{1} e^{-2 \alpha k_{1}} S_{i}
\end{array}\right]<0
\end{aligned}
$$

and

$$
-\alpha \delta+(\varepsilon-\alpha)(\omega-\delta)<0,
$$

$$
\begin{aligned}
& i=2, \ldots, N \text {, where } \\
& \Pi_{i}=\left[\begin{array}{ccccc}
\Pi_{i 11} & \Pi_{i 12} & \Pi_{i 13} & \Pi_{i 14} & \Pi_{i 15} \\
* & \Pi_{i 22} & 0 & \Pi_{i 24} & 0 \\
* & * & \Pi_{i 33} & \Pi_{i 34} & 0 \\
* & * & * & \Pi_{i 44} & \Pi_{i 45} \\
* & * & * & * & \Pi_{i 55}
\end{array}\right], \\
& \tilde{\Pi}_{i}=\left[\begin{array}{ccccc}
\tilde{\Pi}_{i 11} & \Pi_{i 12} & \Pi_{i 13} & \Pi_{i 14} & \Pi_{i 15} \\
* & \Pi_{i 22} & 0 & \Pi_{i 24} & 0 \\
* & * & \Pi_{i 33} & \Pi_{i 34} & 0 \\
* & * & * & \Pi_{i 44} & \Pi_{i 45} \\
* & * & * & * & \Pi_{i 55}
\end{array}\right] \text {, } \\
& \Pi_{i 11}=P_{i}^{T}\left(\tilde{A}_{i}+\alpha I\right)+\left(\tilde{A}_{i}+\alpha I\right)^{T} P_{i}-D_{4 i} L_{i}-L_{i}^{T} D_{4 i}^{T}+3 e^{2 \alpha d} D_{5 i}^{T} T_{i} D_{5 i} \\
& +2 k_{2} e^{2 \alpha k_{2}} D_{6 i}^{T} W_{i} D_{6 i}+2 Q_{i}+k_{1} S_{i}-0.5 e^{-2 \alpha h_{1}} R_{i}-0.5 e^{-2 \alpha h_{2}} R_{i}, \\
& \tilde{\Pi}_{i 11}=P_{i}^{T}\left(\tilde{A}_{i}+\alpha I\right)+\left(\tilde{A}_{i}+\alpha I\right)^{T} P_{i}+2 Q_{i}+k_{1} S_{i}-0.5 e^{-2 \alpha h_{1}} R_{i}-0.5 e^{-2 \alpha h_{2}} R_{i}, \\
& \Pi_{i 12}=P_{i} \tilde{A}_{i}^{T}, \\
& \Pi_{i 13}=e^{-2 \alpha h_{1}} R_{i}, \\
& \Pi_{i 14}=\tilde{B}_{i} P_{i} \\
& \Pi_{i 15}=e^{-2 \alpha h_{2}} R_{i}, \\
& \Pi_{i 22}=h_{1}^{2} R_{i}+h_{2}^{2} R_{i}+\eta^{2} U_{i}-1.5 P_{i},
\end{aligned}
$$




$$
\begin{aligned}
& \Pi_{i 24}=\tilde{B}_{i} P_{i}, \\
& \Pi_{i 33}=-e^{-2 \alpha h_{1}} Q_{i}-e^{-2 \alpha h_{1}} R_{i}-e^{-2 \alpha h_{2}} U_{i}, \\
& \Pi_{i 34}=e^{-2 \alpha h_{2}} U_{i}, \\
& \Pi_{i 44}=-2 e^{-2 \alpha h_{2}} U_{i}, \\
& \Pi_{i 45}=e^{-2 \alpha h_{2}} U_{i}, \\
& \Pi_{i 55}=-2 e^{-2 \alpha h_{2}} U_{i}-2 e^{-2 \alpha h_{2}} Q_{i}-2 e^{-2 \alpha h_{2}} R_{i},
\end{aligned}
$$

then the dynamical networks (7) have exponential synchronization. Moreover, the feedback control is

$$
u_{i}(t)= \begin{cases}-L_{i} P_{i}^{-1} z_{i}(t), & n \omega \leq t \leq n \omega+\delta \\ 0, & n \omega+\delta<t \leq(n+1) \omega .\end{cases}
$$

Proof Case I: for $n \omega \leq t \leq n \omega+\delta$, we choose the Lyapunov-Krasovskii functional as in (17) and using the feedback control (44), we may proof this case by using a similar argument as in the proof of Theorem 3.3. By replacing $D_{1 i}, D_{2 i}$ and $D_{3 i}$ in (12)-(15) with $D_{4 i}, D_{5 i}$, and $D_{6 i}$, respectively. We have

$$
\begin{aligned}
\dot{V}_{i}\left(z_{i}(t)\right)+2 \alpha V_{i}\left(z_{i}(t)\right) \leq & \xi_{i}^{T}(t)\left[(1-\beta) \Pi_{1 i}+\beta \Pi_{2 i}\right] \xi_{i}(t)+y_{i}^{T}(t) \mathcal{N}_{3 i} y_{i}(t) \\
& +\dot{y}_{i}^{T}(t) \mathcal{N}_{4 i} \dot{y}_{i}(t)
\end{aligned}
$$

where $\Pi_{1 i}$ and $\Pi_{2 i}$ are defined as in (35) and (36), respectively, and

$$
\begin{aligned}
& \xi_{i}^{T}(t)=\left[\begin{array}{lllll}
y_{i}^{T}(t) & \dot{y}_{i}^{T}(t) & y_{i}^{T}\left(t-h_{1}\right) & y_{i}^{T}(t-h(t)) & y_{i}^{T}\left(t-h_{2}\right)
\end{array}\right], \\
& \mathcal{N}_{3 i}=-0.5\left(e^{-2 \alpha h_{1}}+e^{-2 \alpha h_{2}}\right) R_{i}+2 k_{1} e^{2 \alpha k_{1}} \tilde{C}_{i} P_{i} S_{i}^{-1} P_{i} \tilde{C}_{i}^{T} \\
& +k_{2} L_{i}^{T} W_{i}^{-1} L_{i}+2 e^{-2 \alpha d} L_{i}^{T} T_{i}^{-1} L_{i}, \\
& \mathcal{N}_{4 i}=-0.5 P_{i}+2 k_{1} e^{2 \alpha k_{1}} \tilde{C}_{i} P_{i} S_{i}^{-1} P_{i} \tilde{C}_{i}^{T}+d^{2} L_{i}^{T} T_{i}^{-1} L_{i}+3 e^{2 \alpha d} D_{5 i}^{T} T_{i}^{-1} D_{5 i} \\
& +2 k_{2} e^{2 \alpha k_{2}} D_{6 i}^{T} W_{i}^{-1} D_{6 i} \text {. }
\end{aligned}
$$

By $(1-\beta) \Pi_{1 i}+\beta \Pi_{2 i}<0$ holds if and only if $\Pi_{1 i}<0$ and $\Pi_{2 i}<0$. Applying the Schur complement lemma, the inequalities $\mathcal{N}_{5 i}<0$ and $\mathcal{N}_{6 i}<0$ are equivalent to $\Pi_{5 i}<0$ and $\Pi_{6 i}<0$, respectively. Therefore, it follows from (35)-(36), (39)-(40), and (45), we obtain

$$
\dot{V}_{i}\left(z_{i}(t)\right)+2 \alpha V_{i}\left(z_{i}(t)\right) \leq 0 \quad \text { for } n \omega \leq t \leq n \omega+\delta .
$$

Thus, by the above differential inequality (46), we have

$$
V_{i}\left(z_{i}(t)\right) \leq V_{i}\left(z_{i}(n \omega)\right) e^{-2 \alpha(t-n \omega)} \quad \text { for } n \omega \leq t \leq n \omega+\delta .
$$

Case II: for $n \omega+\delta \leq t \leq(n+1) \omega$, we choose the Lyapunov-Krasovskii functional having the following form:

$$
V_{i}\left(z_{i}(t)\right)=V_{i 1}(t)+V_{i 2}(t)+V_{i 3}(t)+V_{i 4}(t)+V_{i 5}(t)+V_{i 6}(t)+V_{i 7}(t),
$$


where $V_{i j}(t), j=1,2, \ldots, 7$ are defined similar in (17). We are able to do a similar estimation as we did for Theorem 3.3, and we have the following:

$$
\begin{aligned}
\dot{V}_{i}\left(z_{i}(t)\right)+2 \alpha V_{i}\left(z_{i}(t)\right) \leq & \xi_{i}^{T}(t)\left[(1-\beta) \Pi_{3 i}+\beta \Pi_{4 i}\right] \xi_{i}(t)+y_{i}^{T}(t) \mathcal{N}_{7 i} y_{i}(t)+\dot{y}_{i}^{T}(t) \mathcal{N}_{8 i} \dot{y}_{i}(t) \\
\leq & \xi_{i}^{T}(t)\left[(1-\beta) \Pi_{3 i}+\beta \Pi_{4 i}\right] \xi_{i}(t)+y_{i}^{T}(t) \mathcal{N}_{7 i} y_{i}(t) \\
& +\dot{y}_{i}^{T}(t) \mathcal{N}_{8 i} \dot{y}_{i}(t)+2 \varepsilon V_{i}\left(z_{i}(t)\right)-2 \varepsilon V_{i 1}(t) \\
= & \xi_{i}^{T}(t)\left[(1-\beta) \Pi_{3 i}+\beta \Pi_{4 i}\right] \xi_{i}(t)+y_{i}^{T}(t) \mathcal{N}_{7 i} y_{i}(t) \\
& +\dot{y}_{i}^{T}(t) \mathcal{N}_{8 i} \dot{y}_{i}(t)+2 \varepsilon V_{i}\left(z_{i}(t)\right)-2 \varepsilon y_{i}^{T}(t) P_{i} y_{i}(t) \\
\dot{V}_{i}\left(z_{i}(t)\right)-2(\varepsilon-\alpha) V_{i}\left(z_{i}(t)\right) \leq & \xi_{i}^{T}(t)\left[(1-\beta) \Pi_{3 i}+\beta \Pi_{4 i}\right] \xi_{i}(t) \\
& +y_{i}^{T}(t)\left(\mathcal{N}_{7 i}-2 \varepsilon P_{i}\right) y_{i}(t)+\dot{y}_{i}^{T}(t) \mathcal{N}_{8 i} \dot{y}_{i}(t)
\end{aligned}
$$

where $\Pi_{1 i}$ and $\Pi_{2 i}$ are defined as in (37) and (38), respectively, and

$$
\begin{aligned}
& \xi_{i}^{T}(t)=\left[\begin{array}{lllll}
y_{i}^{T}(t) & \dot{y}_{i}^{T}(t) & y_{i}^{T}\left(t-h_{1}\right) & y_{i}^{T}(t-h(t)) & y_{i}^{T}\left(t-h_{2}\right)
\end{array}\right], \\
& \mathcal{N}_{7 i}=-0.1\left(e^{-2 \alpha h_{1}}+e^{-2 \alpha h_{2}}\right) R_{i}+2 k_{1} e^{2 \alpha k_{1}} \tilde{C}_{i} P_{i} S_{i}^{-1} P_{i} \tilde{C}_{i}^{T}, \\
& \mathcal{N}_{8 i}=-0.5 P_{i}+2 k_{1} e^{2 \alpha k_{1}} \tilde{C}_{i} P_{i} S_{i}^{-1} P_{i} \tilde{C}_{i}^{T} .
\end{aligned}
$$

Now $(1-\beta) \Pi_{3 i}+\beta \Pi_{4 i}<0$ holds if and only if $\Pi_{3 i}<0$ and $\Pi_{4 i}<0$. Applying the Schur complement lemma, the inequalities $\left(\mathcal{N}_{7 i}-2 \varepsilon P_{i}\right)<0$ and $\mathcal{N}_{8 i}<0$ are equivalent to $\Pi_{7 i}<0$ and $\Pi_{8 i}<0$, respectively. Therefore, it follows from (37)-(38), (41)-(42), and (48), that we obtain

$$
\dot{V}_{i}\left(z_{i}(t)\right)-2(\varepsilon-\alpha) V_{i}\left(z_{i}(t)\right) \leq 0 \quad \text { for } n \omega+\delta<t \leq(n+1) \omega .
$$

From the above differential inequality (49), we have

$$
V_{i}\left(z_{i}(t)\right) \leq V_{i}\left(z_{i}(n \omega+\delta)\right) e^{2(\varepsilon-\alpha)(t-n \omega-\delta)} \quad \text { for } n \omega+\delta<t \leq(n+1) \omega .
$$

By (47) and (50), we have

$$
\begin{aligned}
V_{i}\left(z_{i}((n+1) \omega)\right) \leq & V_{i}\left(z_{i}(n \omega+\delta)\right) e^{2(\varepsilon-\alpha)(\omega-\delta)} \\
\leq & V_{i}\left(z_{i}(n \omega)\right) e^{-2 \alpha \delta} e^{2(\varepsilon-\alpha)(\omega-\delta)} \\
& =V_{i}\left(z_{i}(n \omega)\right) e^{-2 \alpha \delta+2(\varepsilon-\alpha)(\omega-\delta)} \\
\leq & V_{i}\left(z_{i}((n-1) \omega+\delta)\right) e^{2 \rho(\omega-\delta)} e^{-2 \alpha \delta+2(\varepsilon-\alpha)(\omega-\delta)} \\
\leq & V_{i}\left(z_{i}((n-1) \omega)\right) e^{-2 \alpha \delta+2(\varepsilon-\alpha)(\omega-\delta)} e^{-2 \alpha \delta+2(\varepsilon-\alpha)(\omega-\delta)} \\
& =V_{i}\left(z_{i}((n-1) \omega)\right) e^{2(-2 \alpha \delta+2(\varepsilon-\alpha)(\omega-\delta))} \\
& \vdots \\
\leq & V_{i}\left(z_{i}(0)\right) e^{(-2 \alpha \delta+2(\varepsilon-\alpha)(\omega-\delta))(n+1)} .
\end{aligned}
$$

For any $t>0$, there is a $n_{0} \geq 0$, such that $n_{0} \omega \leq t \leq\left(n_{0}+1\right) \omega$. 
Case 1. For $n_{0} \omega+\delta \leq t \leq\left(n_{0}+1\right) \omega$, using condition (43), we have

$$
\begin{aligned}
V_{i}\left(z_{i}(t)\right) & \leq V_{i}\left(z_{i}\left(n_{0} \omega+\delta\right)\right) e^{2(\varepsilon-\alpha)\left(t-\left(n_{0} \omega+\delta\right)\right)} \\
& \leq V_{i}\left(z_{i}\left(n_{0} \omega\right)\right) e^{-2 \alpha \delta} e^{2(\varepsilon-\alpha)\left(t-\left(n_{0} \omega+\delta\right)\right)} \\
& \leq V_{i}\left(z_{i}(0)\right) e^{(-2 \alpha \delta+2(\varepsilon-\alpha)(\omega-\delta)) n_{0}} e^{-2 \alpha \delta} e^{2(\varepsilon-\alpha)\left(t-\left(n_{0} \omega+\delta\right)\right)} \\
& \leq V_{i}\left(z_{i}(0)\right) e^{(-2 \alpha \delta+2(\varepsilon-\alpha)(\omega-\delta)) n_{0}} e^{-2 \alpha \delta} e^{2(\varepsilon-\alpha)\left(\left(n_{0}+1\right) \omega-\left(n_{0} \omega+\delta\right)\right)} \\
& =V_{i}\left(z_{i}(0)\right) e^{(-2 \alpha \delta+2(\varepsilon-\alpha)(\omega-\delta))\left(n_{0}+1\right)} \\
& =V_{i}\left(z_{i}(0)\right) e^{\frac{(-2 \alpha \delta+2(\varepsilon-\alpha)(\omega-\delta))\left(n_{0}+1\right) \omega}{\omega}} \\
& \leq V_{i}\left(z_{i}(0)\right) e^{\frac{(-2 \alpha \delta+2(\varepsilon-\alpha)(\omega-\delta)) t}{\omega}}
\end{aligned}
$$

Case 2. For $n_{0} \omega \leq t \leq n_{0} \omega+\delta$, using condition (43), we have

$$
\begin{aligned}
V_{i}\left(z_{i}(t)\right) & \leq V_{i}\left(z_{i}\left(n_{0} \omega\right)\right) e^{-2 \alpha\left(t-n_{0} \omega\right)} \\
& \leq V_{i}\left(z_{i}(0)\right) e^{(-2 \alpha \delta+2(\varepsilon-\alpha)(\omega-\delta)) n_{0}} e^{-2 \alpha\left(t-n_{0} \omega\right)} \\
& \leq V_{i}\left(z_{i}(0)\right) e^{(-2 \alpha \delta+2(\varepsilon-\alpha)(\omega-\delta)) n_{0}} \\
& =V_{i}\left(z_{i}(0)\right) e^{-(-2 \alpha \delta+2(\varepsilon-\alpha)(\omega-\delta))} e^{(-2 \alpha \delta+2(\varepsilon-\alpha)(\omega-\delta))\left(n_{0}+1\right)} \\
& =V_{i}\left(z_{i}(0)\right) e^{-(-2 \alpha \delta+2(\varepsilon-\alpha)(\omega-\delta))} e^{\frac{(-2 \alpha \delta+2(\varepsilon-\alpha)(\omega-\delta))\left(n_{0}+1\right) \omega}{\omega}} \\
& \leq V_{i}\left(z_{i}(0)\right) e^{-(-2 \alpha \delta+2(\varepsilon-\alpha)(\omega-\delta))} e^{\frac{(-2 \alpha \delta+2(\varepsilon-\alpha)(\omega-\delta)) t}{\omega}}
\end{aligned}
$$

Let $\xi=e^{-(-2 \alpha \delta+2(\varepsilon-\alpha)(\omega-\delta))}$. By (51) and (52), we have

$$
V_{i}\left(z_{i}(t)\right) \leq \xi V_{i}\left(z_{i}(0)\right) e^{\frac{(-2 \alpha \delta+2(\varepsilon-\alpha)(\omega-\delta)) t}{\omega}}, \quad \forall t \geq 0
$$

On the other hand, using the condition (18), we have obtained the following:

$$
\left\|z_{i}(t)\right\| \leq \sqrt{\frac{\mathcal{N} \xi}{\gamma}} e^{\frac{(-\alpha \delta+(\varepsilon-\alpha)(\omega-\delta)) t}{\omega}}, \quad \forall t \geq 0
$$

which implies the dynamical networks (7) is exponentially stable under the controller $\mathrm{H} 2$, then exponential synchronization of the controlled dynamical networks (5) is achieved. The proof is thus completed.

Remark 3.5 It is clear that as $\delta \rightarrow \omega$ the intermittent feedback control will reduce to a continuous feedback. In this case, presented in Theorem 3.3.

Remark 3.6 In [14-16], the authors investigated synchronization of complex dynamical network with coupling time-delay, but the time-delay considered in these three works are assumed to be constants delay. In [9], Li et al. presented synchronization in complex dynamical networks with time-varying delays in the network couplings and time-varying delays in the dynamical nodes, but the time-varying delays are required to be differentiable, which is a very strict condition. Obviously, we do not need these limit condition in this paper. 
Remark 3.7 If $k_{1}(t)=0, c_{1}=0, c_{3}=0$, and $\mathcal{U}_{i}(t)=0$, then system (1) reduces to the following system presented in $[9,18]$ :

$$
\dot{x}_{i}(t)=f\left(x_{i}(t)\right)+c_{2} \sum_{j=1}^{N} b_{i j} G_{2} x_{j}(t-h(t)), \quad t>0, i=1,2, \ldots, N .
$$

According to Theorem 3.3, we obtain the following corollary for the synchronization of network (53).

Corollary 3.8 For some given scalars $0<\alpha$, the dynamical networks (53) with time-varying delay $h(t)$ satisfying (6) are exponentially synchronization if there exist symmetric positive definite matrices $P_{i}>0, Q_{i}>0, R_{i}>0, U_{i}>0$, such that the following symmetric linear matrix inequality holds:

$$
\begin{aligned}
& \Gamma_{i 1}=\Gamma_{i}-\left[\begin{array}{lllll}
0 & 0 & I & -I & 0
\end{array}\right]^{T} e^{-2 \alpha h_{2}} U_{i}\left[\begin{array}{ccccc}
0 & 0 & I & -I & 0
\end{array}\right]<0, \\
& \Gamma_{i 2}=\Gamma_{i}-\left[\begin{array}{lllllll}
0 & 0 & 0 & I & -I
\end{array}\right]^{T} e^{-2 \alpha h_{2}} U_{i}\left[\begin{array}{lllll}
0 & 0 & 0 & I & -I
\end{array}\right]<0,
\end{aligned}
$$

where

$$
\begin{aligned}
& \Gamma_{i 11}=P_{i}^{T}(J(t)+\alpha I)+(J(t)+\alpha I)^{T} P_{i}+2 Q_{i}-e^{-2 \alpha h_{1}} R_{i}-e^{-2 \alpha h_{2}} R_{i}, \\
& \Gamma_{i 12}=P_{i} J^{T}(t) \\
& \Gamma_{i 13}=e^{-2 \alpha h_{1}} R_{i} \\
& \Gamma_{i 14}=c_{2} \lambda_{2 i} G_{2} P_{i} \\
& \Gamma_{i 15}=e^{-2 \alpha h_{2}} R_{i} \\
& \Gamma_{i 22}=h_{1}^{2} R_{i}+h_{2}^{2} R_{i}+\eta^{2} U_{i}-2 P_{i} \\
& \Gamma_{i 24}=c_{2} \lambda_{2 i} G_{2} P_{i} \\
& \Gamma_{i 33}=-e^{-2 \alpha h_{1}} Q_{i}-e^{-2 \alpha h_{1}} R_{i}-e^{-2 \alpha h_{2}} U_{i} \\
& \Gamma_{i 34}=e^{-2 \alpha h_{2}} U_{i} \\
& \Gamma_{i 44}=-2 e^{-2 \alpha h_{2}} U_{i} \\
& \Gamma_{i 45}=e^{-2 \alpha h_{2}} U_{i} \\
& \Gamma_{i 55}=-2 e^{-2 \alpha h_{2}} U_{i}-2 e^{-2 \alpha h_{2}} Q_{i}-2 e^{-2 \alpha h_{2}} R_{i} .
\end{aligned}
$$

Proof Similar to proof of Theorem 3.3. Indeed, by setting $S_{i}=0, T_{i}=0$, and $W_{i}=0$ in (17), one may easily derive the result and hence the proof is omitted.

Remark 3.9 In [31-34], the authors investigated synchronization of complex dynamical network with coupling time-delay based on intermittent control, but the controller is presented in terms of nominal state-delayed systems. On the other hands, we have considered more complicated problem, namely, synchronization of complex dynamical network with hybrid coupling delay and mixed time-varying delay (interval time-varying delay and distributed time-varying delay), which time-varying delay using both state-delayed feedback control as well as intermittent state-delayed feedback control. It should be pointed out that the synchronization problem for complex dynamical networks with both interval 
and distributed time-varying delays has not received much attention in the literature, not to mention the case when the coupling and controller are also involved.

\section{Numerical examples}

In this section, we now provide an example to show the effectiveness of the result in Theorem 3.3 and Theorem 3.4.

Example 4.1 We first consider the perturbed Chua circuit system with mixed timevarying delays is used as uncoupled node in the network (1) to show the effectiveness of the proposed control scheme. The perturbed Chua circuit system with mixed timevarying delays is given by [43]

$$
\begin{aligned}
& \dot{x}_{1}(t)=p\left(x_{2}(t-h(t))-\frac{1}{7}\left(2 x_{1}^{3}(t)-x_{1}(t)\right)\right), \\
& \dot{x}_{2}(t)=x_{1}(t)-s x_{2}(t)+x_{3}(t-h(t)), \\
& \dot{x}_{3}(t)=q x_{2}(t)+r \int_{t-k_{1}(t)}^{t} x_{1}^{2}(s) d s,
\end{aligned}
$$

where $p, q, r$, and $s$ are real positive constants. It is well known that the system (56) exhibits chaotic behavior with the parameters $p, q, r$, and $s$ are chosen as $p=7, q=-\frac{100}{7}, r=0.07$, and $s=1.5$, the initial condition function $\phi(t)=[0.65 \cos t, \sin t, \sin t]^{T}$, the time-varying delay functions $h(t)=0.1+0.1|\sin t|$ and $k_{1}(t)=0.1|\cos t|$ is shown in Figure 1 . The solution of the system (56) is denoted by $s(t)=\left(s_{1}(t), s_{2}(t), s_{3}(t)\right)^{T}$, which is shown in Figure 2 . It is stable at the equilibrium point $s(t)=0, s(t-h(t))=0, \int_{t-k_{1}(t)}^{t} s(\theta) d \theta=0$, and the Jacobian matrices are

$$
J(t)=\left[\begin{array}{ccc}
1 & 0 & 0 \\
1 & -1.5 & 0 \\
0 & -\frac{100}{7} & 0
\end{array}\right], \quad J_{h}(t)=\left[\begin{array}{ccc}
0 & 7 & 0 \\
0 & 0 & 1 \\
0 & 0 & 0
\end{array}\right], \quad J_{k_{1}}(t)=\left[\begin{array}{ccc}
0 & 0 & 0 \\
0 & 0 & 0 \\
0 & 0 & 0
\end{array}\right] .
$$

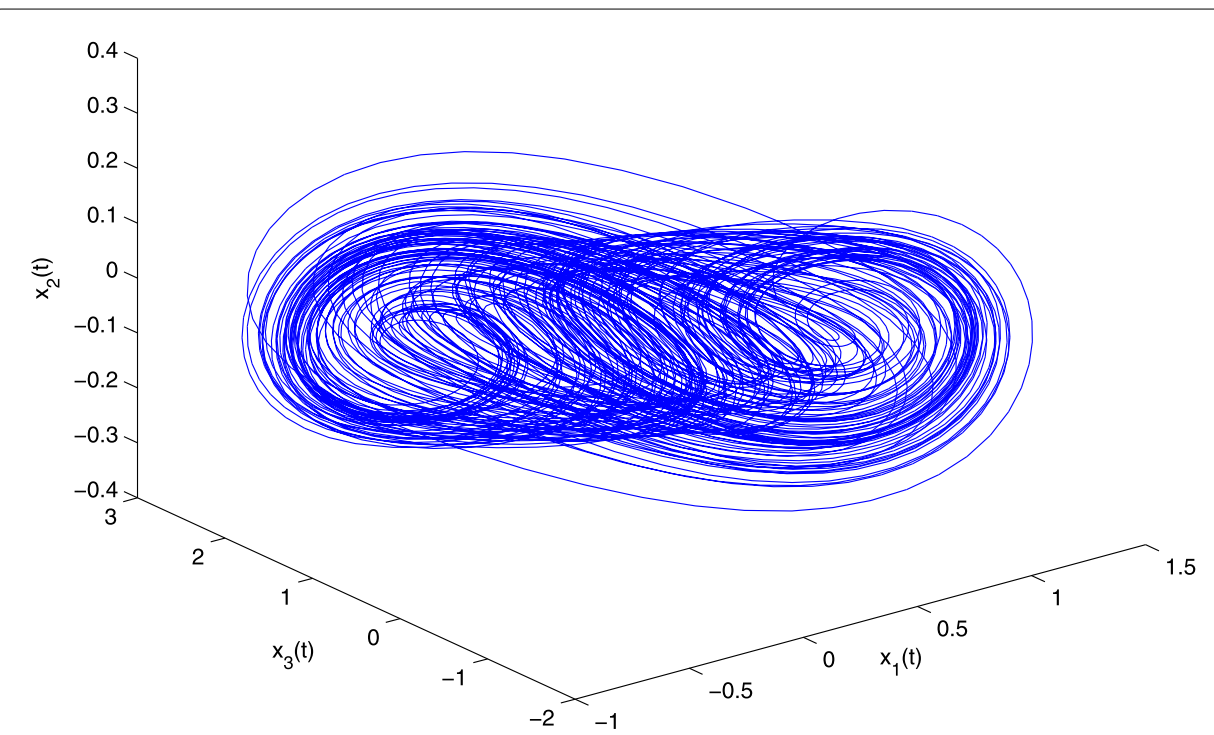

Figure 1 Chaotic behavior of the perturbed Chua circuit system with mixed time-varying delays (56). 


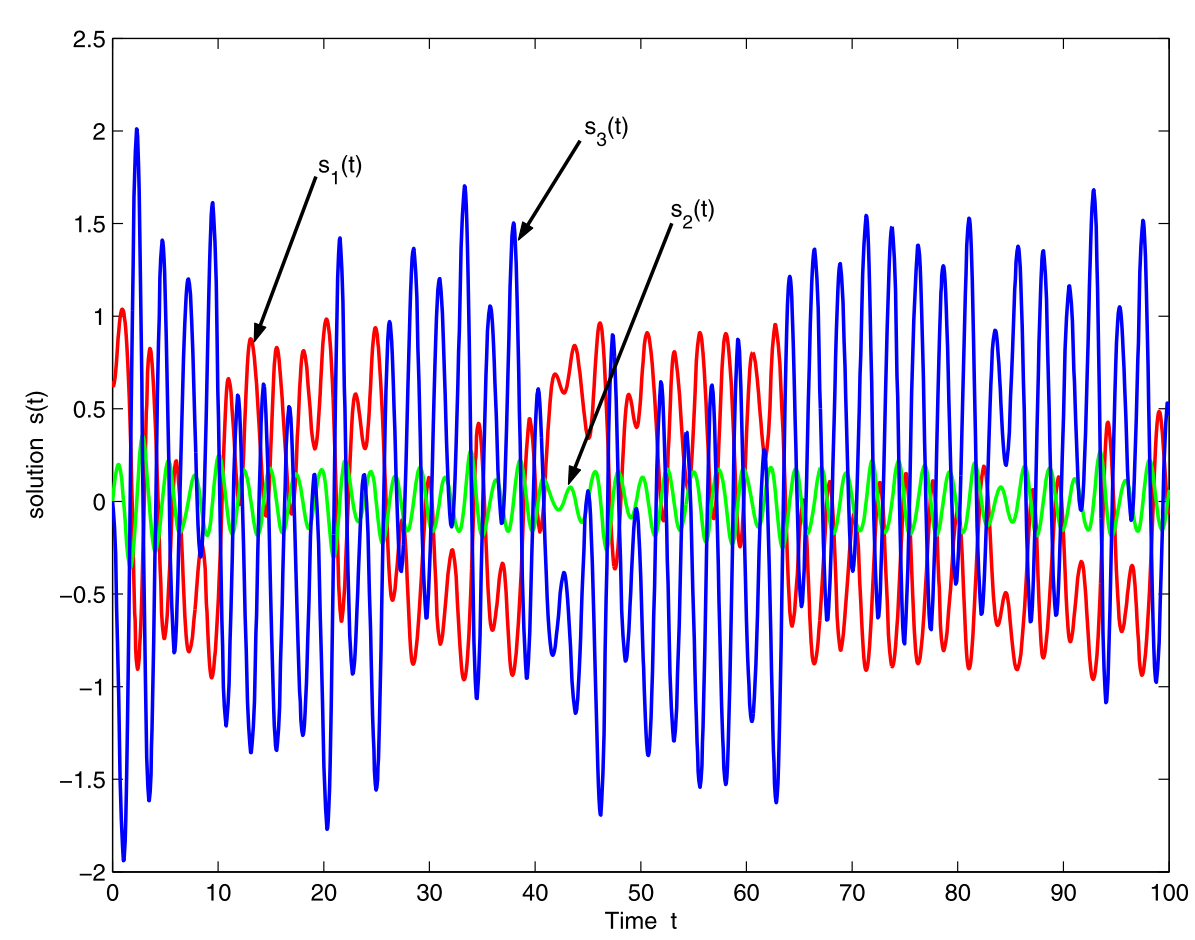

Figure 2 Solution $s(t)$ of the perturbed Chua circuit system with mixed time-varying delays (56).

Consider a network consisting of five identical perturbed Chua circuit system with mixed time-varying and hybrid coupling delays. The corresponding controlled dynamical network (4) can be described as

$$
\begin{aligned}
\dot{x}_{i}(t)= & f\left(x_{i}(t), x_{i}(t-h(t)), \int_{t-k_{1}(t)}^{t} x_{i}(s) d s\right)+c_{1} \sum_{j=1}^{N} a_{i j} G_{1} x_{j}(t) \\
& +c_{2} \sum_{j=1}^{N} b_{i j} G_{2} x_{j}(t-h(t))+c_{3} \sum_{j=1}^{N} c_{i j} G_{3} \int_{t-k_{1}(t)}^{t} x_{j}(s) d s \\
& +D_{1 i} K_{i}\left(x_{i}(t)-s(t)\right)+D_{2 i} K_{i}\left(x_{i}(t-d(t))-s(t-d(t))\right) \\
& +D_{3 i} K_{i}\left(\int_{t-k_{2}(t)}^{t} x_{i}(s) d s-\int_{t-k_{2}(t)}^{t} s(\theta) d \theta\right), \quad i=1,2, \ldots, 5 .
\end{aligned}
$$

Assume that $D_{1 i}=\operatorname{diag}\{3,3,3\}, D_{2 i}=\operatorname{diag}\{0.1,0.1,0.1\}, D_{3 i}=\operatorname{diag}\{0.1,0.1,0.1\}, i=1,2$, $\ldots, 5$, the coupling strength $c_{1}=0.3, c_{2}=0.2, c_{3}=0.4$, the inner-coupling matrices are

$$
\begin{aligned}
G_{1} & =\left[\begin{array}{lll}
2 & 0 & 0 \\
0 & 2 & 0 \\
0 & 0 & 2
\end{array}\right], \quad G_{2}=\left[\begin{array}{lll}
1 & 0 & 0 \\
0 & 1 & 0 \\
0 & 0 & 1
\end{array}\right], \\
G_{3} & =\left[\begin{array}{ccc}
0.5 & 0 & 0 \\
0 & 0.5 & 0 \\
0 & 0 & 0.5
\end{array}\right],
\end{aligned}
$$



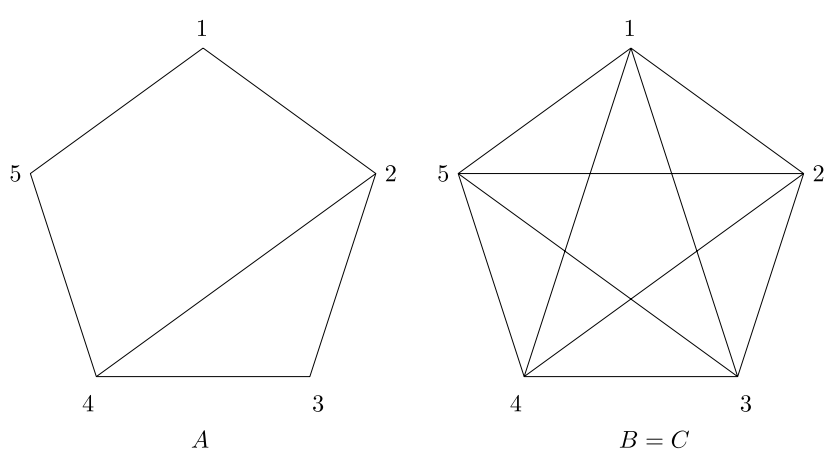

Figure 3 The topology structure of complex networks with $N=5$.

and the outer-coupling matrices are given by the following irreducible symmetric matrices satisfying condition (2):

$$
A=\left[\begin{array}{ccccc}
-2 & 1 & 0 & 0 & 1 \\
1 & -3 & 1 & 1 & 0 \\
0 & 1 & -2 & 1 & 0 \\
0 & 1 & 1 & -3 & 1 \\
1 & 0 & 0 & 1 & -2
\end{array}\right], \quad B=C=\left[\begin{array}{ccccc}
-4 & 1 & 1 & 1 & 1 \\
1 & -4 & 1 & 1 & 1 \\
1 & 1 & -4 & 1 & 1 \\
1 & 1 & 1 & -4 & 1 \\
1 & 1 & 1 & 1 & -4
\end{array}\right]
$$

and the topology structure of complex networks is shown in Figure 3.

The eigenvalues of $A, B$, and $C$ are $\lambda_{1}=\{0,-1.382,-2.382,-3.618,-4.618\}, \lambda_{2}=\{0,-5$, $-5,-5,-5\}$, and $\lambda_{3}=\{0,-5,-5,-5,-5\}$, respectively.

Solution: From the conditions (12)-(15) of Theorem 3.3, we let $\alpha=0.02, h_{1}=0.1, h_{2}=0.2$, $k_{1}=0.1, k_{2}=0.1, d=0.3$, the gain matrices of the desired controllers can be obtained as follows:

$$
\begin{array}{ll}
K_{1}=\left[\begin{array}{ccc}
-4.6038 & -0.2266 & -0.8478 \\
-0.1812 & -15.2461 & 2.5773 \\
-1.0007 & 3.6578 & -4.2867
\end{array}\right], \quad K_{2}=\left[\begin{array}{ccc}
-3.8398 & -0.1242 & -1.0323 \\
-0.0015 & -15.0343 & 1.9854 \\
-1.0438 & 2.7597 & -4.1461
\end{array}\right], \\
K_{3}=\left[\begin{array}{ccc}
-3.7311 & 0.0828 & -1.2030 \\
0.2016 & -14.8969 & 1.8453 \\
-1.1970 & 2.4629 & -4.2466
\end{array}\right], \quad K_{4}=\left[\begin{array}{ccc}
-3.9213 & 0.3286 & -1.4385 \\
0.5011 & -15.4924 & 1.7291 \\
-1.4346 & 2.1419 & -4.6878
\end{array}\right], \\
K_{5}=\left[\begin{array}{ccc}
-4.3268 & 0.5100 & -1.6409 \\
0.7973 & -16.7178 & 1.6821 \\
-1.6546 & 1.9279 & -5.3012
\end{array}\right] .
\end{array}
$$

The numerical simulations are carried out using the explicit Runge-Kutta-like method (dde45), interpolation and extrapolation by spline of the third order. Figure 4 shows the synchronization between the states of isolate node $s(t)$ and node $x_{i}(t), i=1,2, \ldots, 5$. Figure 5 shows the synchronization errors between the states of isolate node $s(t)$ and node $x_{i}(t)$, where $e_{i j}(t)=x_{i j}(t)-s_{i}(t)$, for $i=1, \ldots, 5, j=1,2,3$, without feedback control. Figure 6 shows the synchronization errors between the states of isolated node $s(t)$ and node $x_{i}(t)$, 

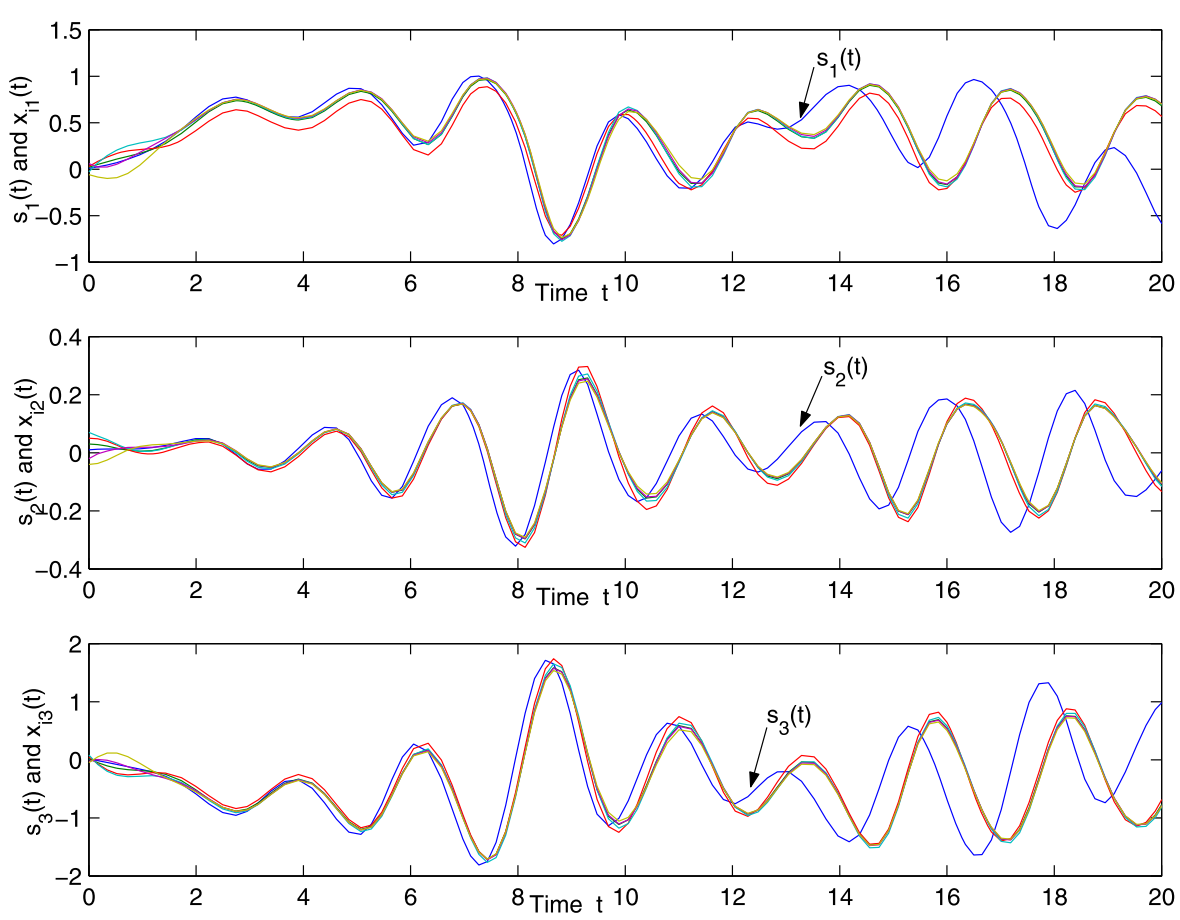

Figure 4 Synchronization curves for the states of the isolated node $s(t)$ and node $x_{i}(t), i=1,2, \ldots, 5$.
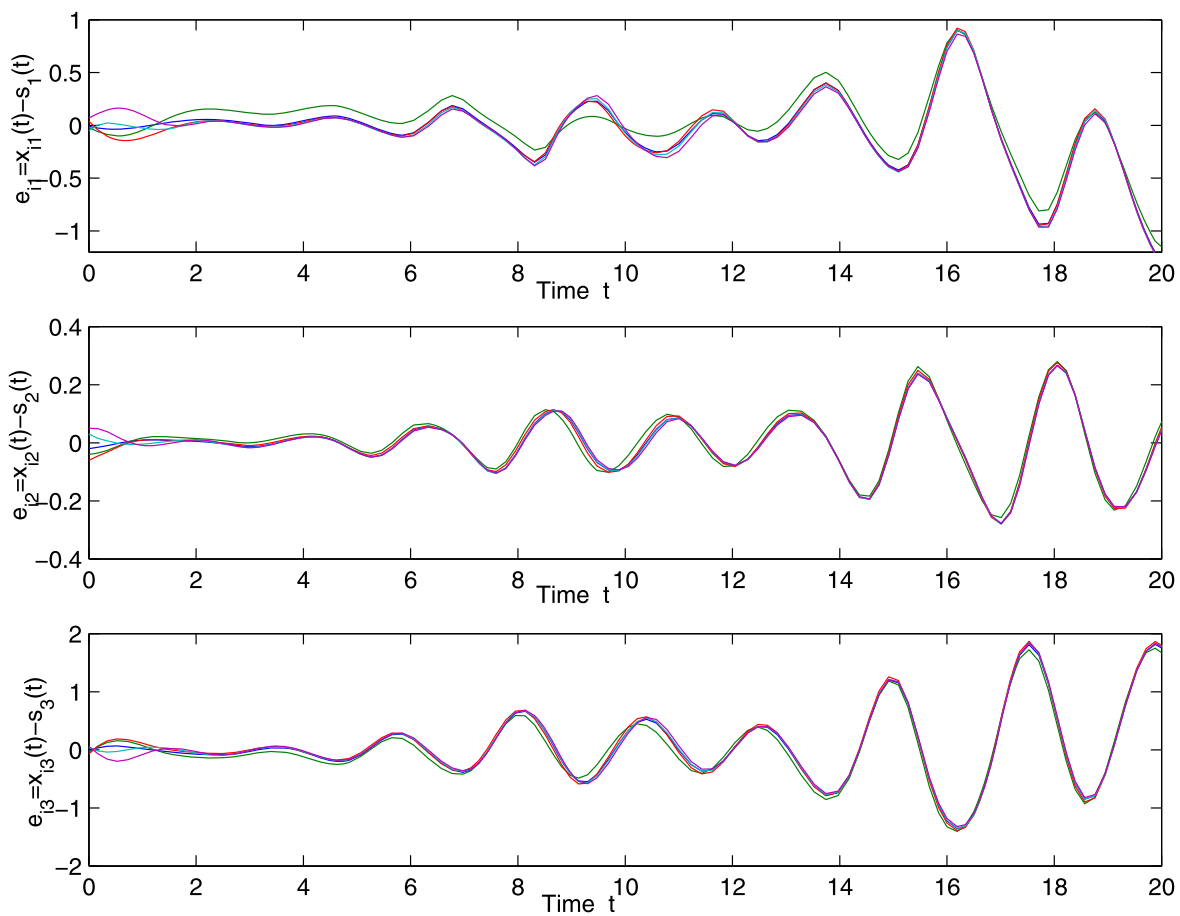

Figure 5 Synchronization error curves for the isolated node $s(t)$ and node $x_{i}(t)$, where $e_{i j}(t)=x_{i j}(t)-s_{i}(t)$, for $i=1, \ldots, 5, j=1,2,3$, without feedback control. 


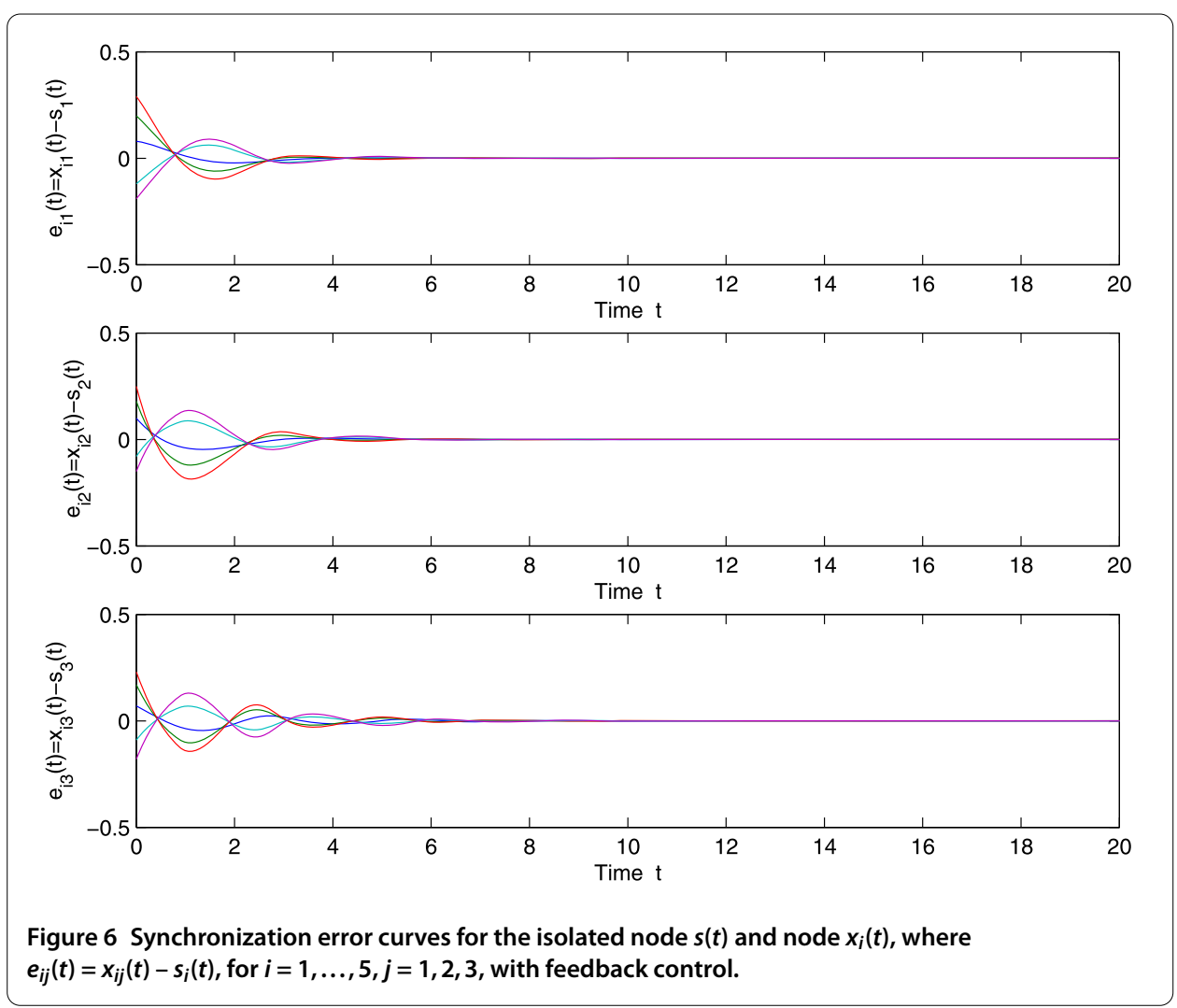

where $e_{i j}(t)=x_{i j}(t)-s_{i}(t)$, for $i=1, \ldots, 5, j=1,2,3$, with feedback control. We see that the synchronization errors converge to zero under the above conditions.

Example 4.2 We consider the nonlinear network model with five nodes, in which each node is a Lorenz system with mixed time-varying delay described by [7]

$$
\begin{aligned}
& \dot{x}_{i 1}(t)=a\left(x_{i 2}(t)-x_{i 1}(t)\right), \\
& \dot{x}_{i 2}(t)=c x_{i 1}(t-h(t))-x_{i 2}(t)-x_{i 1}(t) x_{i 3}(t-h(t)), \\
& \dot{x}_{i 3}(t)=x_{i 1}(t) \int_{t-k_{1}(t)}^{t} x_{i 2}(s) d s-b x_{i 3}(t-h(t)),
\end{aligned}
$$

where $a=0.9, b=1.3$, and $c=-1$. For the initial function $\phi(t)=[-3.2 \cos t, 2 \cos t, 5 \cos t]$ the solution of system (57) is denoted by $s(t)=\left(s_{1}(t), s_{2}(t), s_{3}(t)\right)^{T}$, which is shown in Figure 7. It is asymptotically stable at the equilibrium point $s(t)=0, s(t-h(t))=0$, $\int_{t-k_{1}(t)}^{t} s(\theta) d \theta=0$ and its Jacobian matrices are

$$
J(t)=\left[\begin{array}{ccc}
-0.9 & 0.9 & 0 \\
0 & -1 & 0 \\
0 & 0 & 0
\end{array}\right], \quad J_{h(t)}=\left[\begin{array}{ccc}
-1 & 0 & 0 \\
0 & 0 & 0 \\
0 & 0 & -1.3
\end{array}\right], \quad J_{k_{1}}(t)=\left[\begin{array}{ccc}
0 & 0 & 0 \\
0 & 0 & 0 \\
0 & 0 & 0
\end{array}\right]
$$

Assume that $D_{4 i}=\operatorname{diag}\{2,2,2\}, D_{5 i}=\operatorname{diag}\{0.1,0.1,0.1\}, D_{6 i}=\operatorname{diag}\{0.1,0.1,0.1\}, i=$ $1,2, \ldots, 5$, the coupling strength $c_{1}=0.1, c_{2}=0.2, c_{3}=0.3$, the inner-coupling matrices 


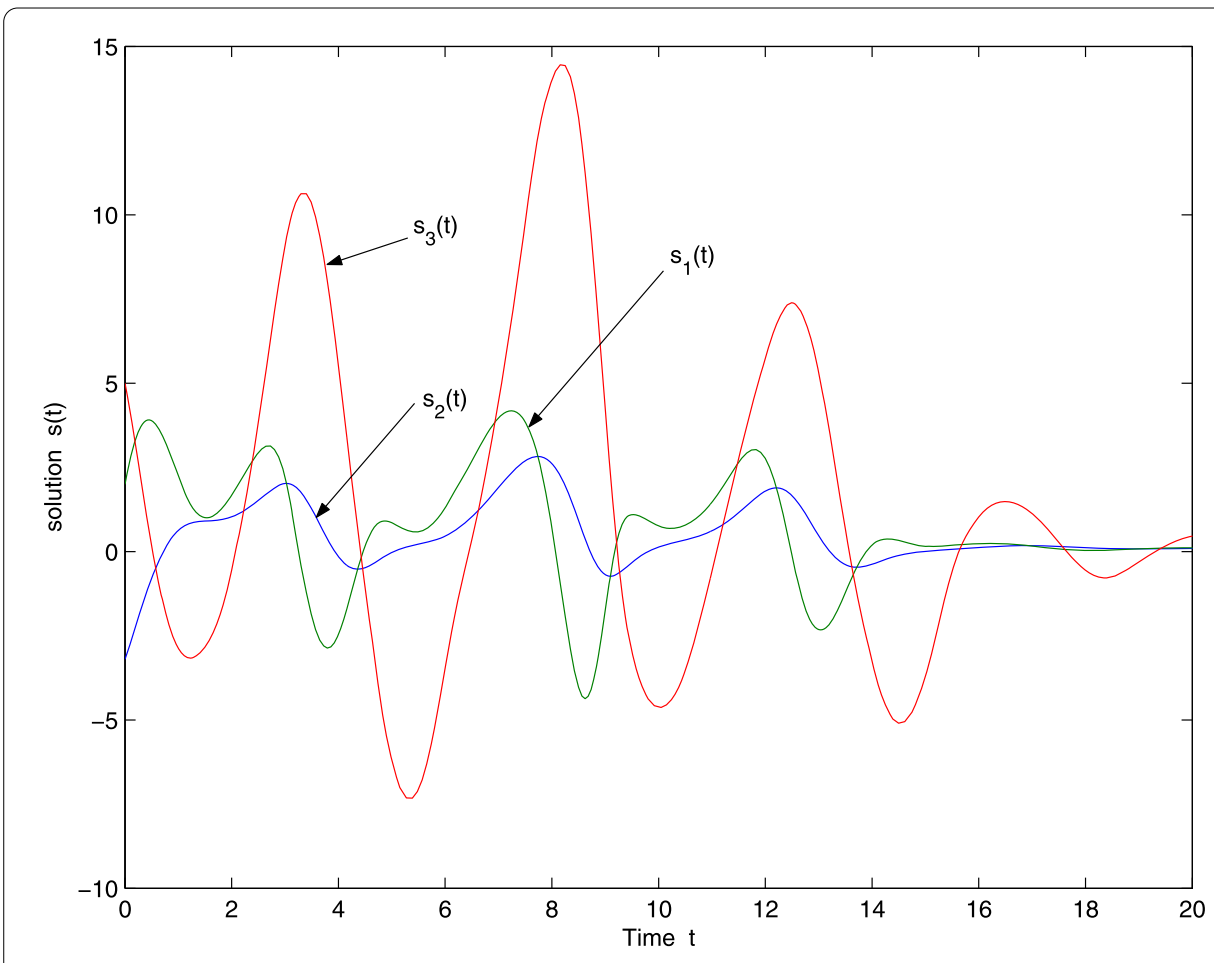

Figure 7 Solution of the Lorenz system with mixed time-varying delays (57).

are

$$
G_{1}=\left[\begin{array}{ccc}
0.4 & 0 & 0 \\
0 & 0.4 & 0 \\
0 & 0 & 0.4
\end{array}\right], \quad G_{2}=\left[\begin{array}{ccc}
0.5 & 0 & 0 \\
0 & 0.5 & 0 \\
0 & 0 & 0.5
\end{array}\right], \quad G_{3}=\left[\begin{array}{ccc}
0.3 & 0 & 0 \\
0 & 0.3 & 0 \\
0 & 0 & 0.3
\end{array}\right]
$$

and the outer-coupling matrices are given by the following irreducible symmetric matrices satisfying condition (2):

$$
A=C=\left[\begin{array}{ccccc}
-4 & 1 & 1 & 1 & 1 \\
1 & -3 & 1 & 1 & 0 \\
1 & 1 & -3 & 0 & 1 \\
1 & 1 & 0 & -2 & 0 \\
1 & 0 & 1 & 0 & -2
\end{array}\right], \quad B=\left[\begin{array}{ccccc}
-4 & 1 & 1 & 1 & 1 \\
1 & -4 & 1 & 1 & 1 \\
1 & 1 & -4 & 1 & 1 \\
1 & 1 & 1 & -4 & 1 \\
1 & 1 & 1 & 1 & -4
\end{array}\right]
$$

and the topology structure of complex networks is shown in Figure 8.

The eigenvalues of $A, B$, and $C$ are $\lambda_{1}=\{0,-1.5858,3,-4.4142,-5\}, \lambda_{2}=\{0,-5,-5,-5$, $-5\}$, and $\lambda_{3}=\{0,-1.5858,3,-4.4142,-5\}$, respectively.

Solution: From the conditions (35)-(43) of Theorem 3.4, we let $\varepsilon=0.09, \alpha=0.07, \omega=$ $4, \delta=2.5, h_{1}=0.1, h_{2}=0.2, k_{1}=0.1, k_{2}=0.12, d=0.3$; the gain matrices of the desired controllers can be obtained as follows:

$$
K_{1}=\left[\begin{array}{ccc}
-0.1592 & -0.0094 & 0 \\
-0.0155 & -0.1306 & 0 \\
0 & 0 & -0.3712
\end{array}\right], \quad K_{2}=\left[\begin{array}{ccc}
-0.2479 & -0.0265 & 0 \\
-0.0336 & -0.2273 & 0 \\
0 & 0 & -0.7249
\end{array}\right] \text {, }
$$



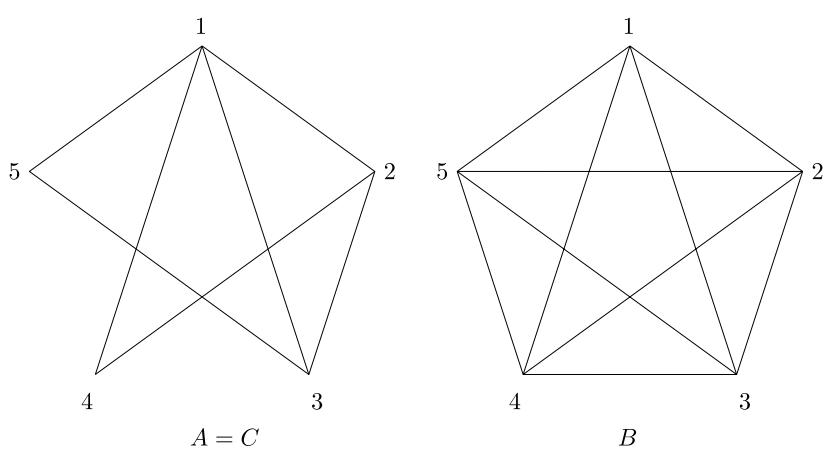

Figure 8 The topology structure of complex networks with $N=5$.
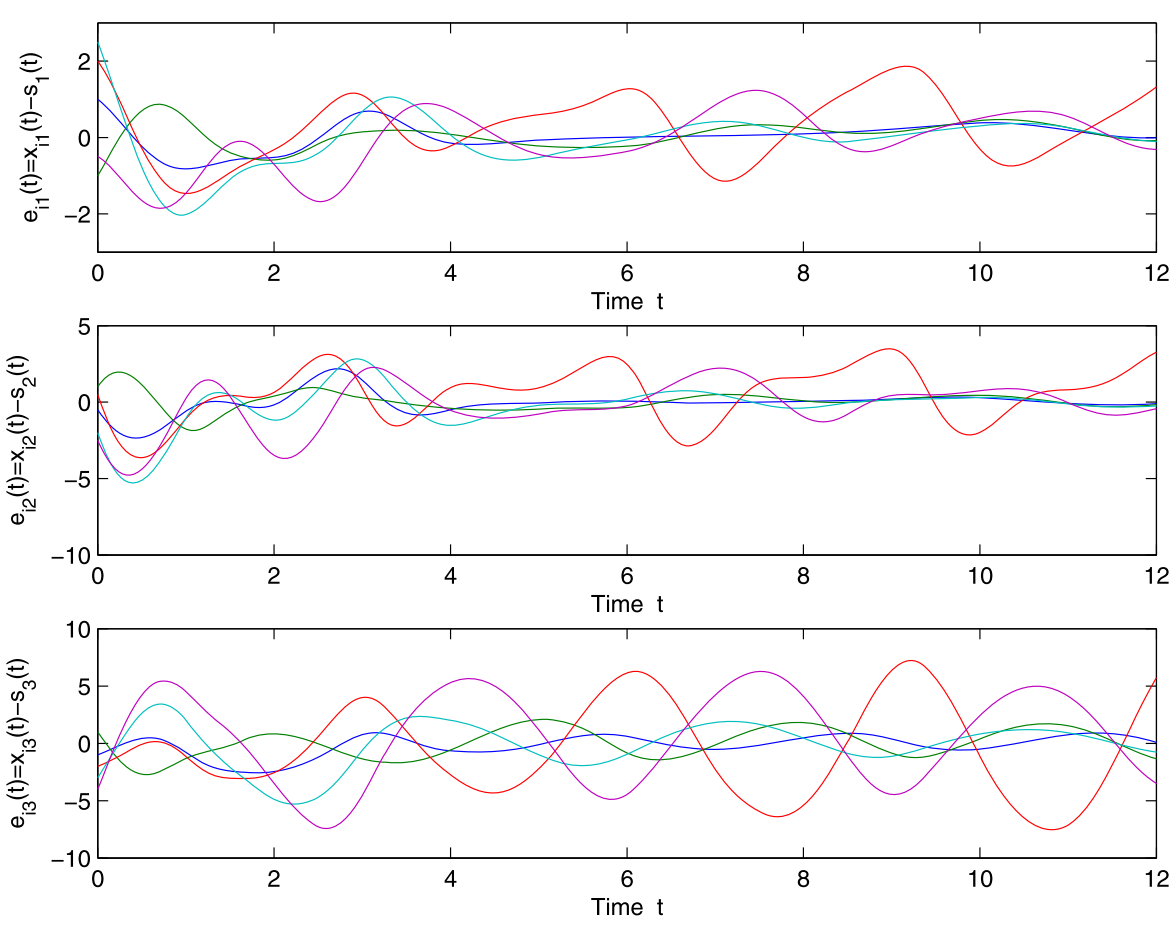

Figure 9 Synchronization error curves for the isolated node $s(t)$ and node $x_{i}(t)$, where $e_{i j}(t)=x_{i j}(t)-s_{i}(t)$, for $i=1, \ldots, 5, j=1,2,3$, without intermittent feedback control.

$$
\begin{aligned}
K_{3} & =\left[\begin{array}{ccc}
-0.2325 & -0.0247 & 0 \\
-0.0306 & -0.2134 & 0 \\
0 & 0 & -0.7079
\end{array}\right], \quad K_{4}=\left[\begin{array}{ccc}
-0.2188 & -0.0234 & 0 \\
-0.0286 & -0.2006 & 0 \\
0 & 0 & -0.6986
\end{array}\right], \\
K_{5} & =\left[\begin{array}{ccc}
-0.2132 & -0.0230 & 0 \\
-0.0279 & -0.1953 & 0 \\
0 & 0 & -0.6962
\end{array}\right] .
\end{aligned}
$$

Figure 9 shows the synchronization errors between the states of the isolated node $s(t)$ and node $x_{i}(t)$, where $e_{i j}(t)=x_{i j}(t)-s_{i}(t)$, for $i=1, \ldots, 5, j=1,2,3$, without intermittent feedback control. Figure 10 shows the synchronization errors between the states of the 

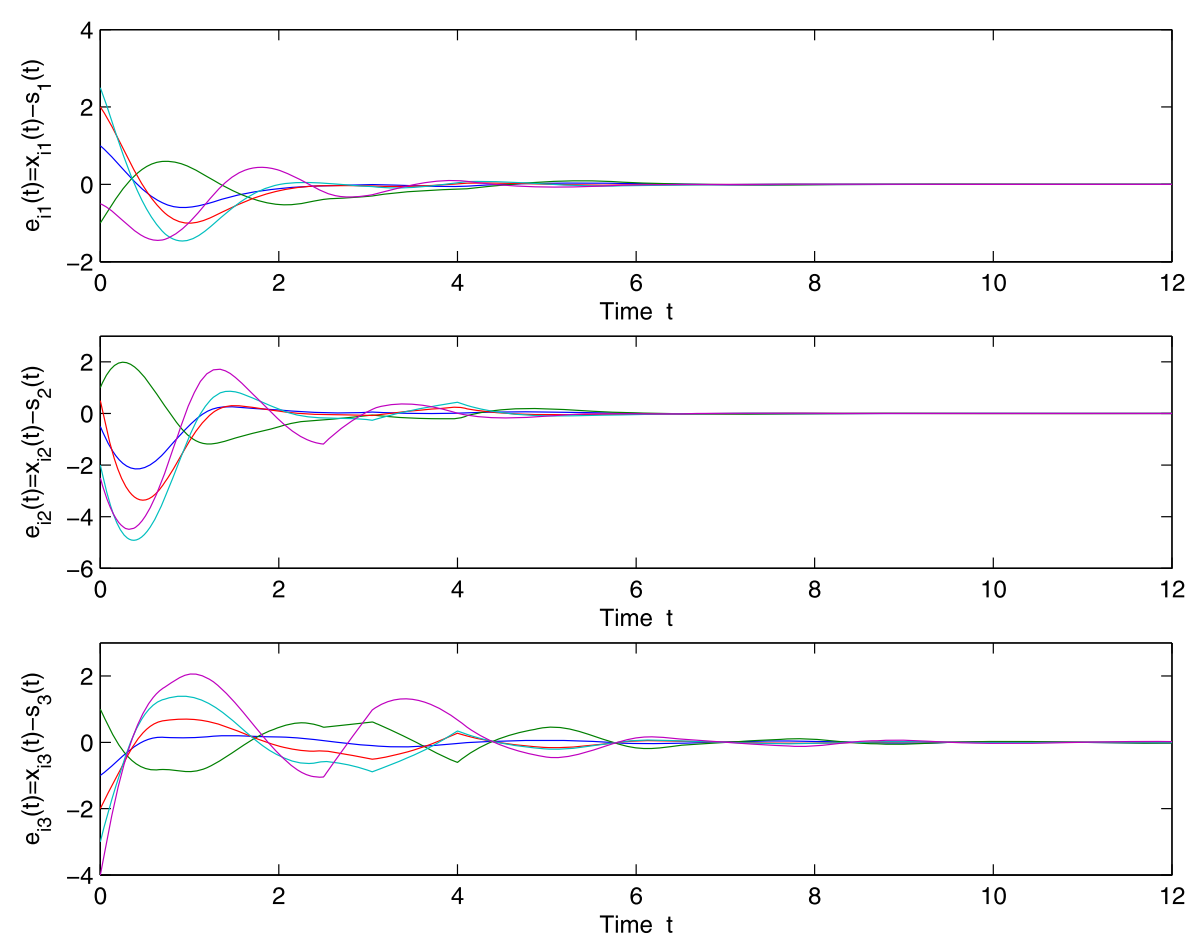

Figure 10 Synchronization error curves for the isolated node $s(t)$ and node $x_{i}(t)$, where $e_{i j}(t)=x_{i j}(t)-s_{i}(t)$, for $i=1, \ldots, 5, j=1,2,3$, with intermittent feedback control.

isolated node $s(t)$ and node $x_{i}(t)$, where $e_{i j}(t)=x_{i j}(t)-s_{i}(t)$, for $i=1, \ldots, 5, j=1,2,3$, with intermittent feedback control. We see that the synchronization errors converge to zero under the above conditions.

Remark 4.1 In Example 4.1 and Example 4.2, each of them to consider general complex networks in which every dynamical node has mixed time-varying delays (interval timevarying delay and distributed time-varying delay), and the complex networks have state coupling, interval time-varying delay coupling and distributed time-varying delay coupling.

Example 4.3 Consider a network model with five nodes, where each node is a threedimensional stable linear system described by $[9,18]$

$$
\begin{aligned}
& \dot{x}_{i 1}(t)=-x_{i 1}(t), \\
& \dot{x}_{i 2}(t)=-2 x_{i 2}(t), \\
& \dot{x}_{i 3}(t)=-3 x_{i 3}(t),
\end{aligned}
$$

which is asymptotically stable at the equilibrium point $s(t)=0$, and its Jacobian matrix is $J(t)=\operatorname{diag}\{-1,-2,-3\}$. Assume that the network coupling is the same as that in Example 4.1. The upper bounds on the time-delay obtained from Corollary 3.8 are listed in Table 1. We see that Corollary 3.8 provides a less conservative result than those obtained via the methods of $[9,18]$. When $h_{m} \neq 0$ especially, the result in [9] is not discussed while Corollary 3.8 in this paper also considers the case $h_{m} \neq 0$. 
Table 1 Comparison of the maximum value $h_{M}\left(h_{m}=0\right)$ for difference $c_{2}$

\begin{tabular}{lllll}
\hline $\mathbf{c}_{\mathbf{2}}$ & $\mathbf{0 . 3}$ & $\mathbf{0 . 4}$ & $\mathbf{0 . 5}$ & $\mathbf{0 . 6}$ \\
\hline Li et al. [9] & 0.960 & 0.710 & 0.562 & 0.464 \\
Yue and Li [18] & 1.345 & 0.950 & 0.731 & 0.587 \\
Corollary 3.8 & 1.9707 & 1.2848 & 0.8712 & 0.5941 \\
\hline
\end{tabular}

Remark 4.2 In [9] presented the synchronization problem of general complex dynamical networks with time-varying delays in the network couplings and time-varying delays in the dynamical nodes, respectively. But the time-varying delays are required to be differentiable, however, in most cases, these conditions are difficult to satisfy. Therefore, in this paper we will employ some new techniques so that the above conditions can be removed.

\section{Conclusions}

This paper has investigated synchronization for complex dynamical network with mixed time-varying and hybrid coupling delays, which is composed of state coupling, interval time-varying delay coupling, and distributed time-varying delay coupling. The timevarying delay function is not necessary to be differentiable which allows the time-delay function to be a fast time-varying function. We transformed the synchronization problem of the complex network into the stability analysis of linear systems. A new class of Lyapunov-Krasovskii functionals is constructed; new delay-dependent sufficient conditions for the exponential synchronization of complex dynamical network have been derived by a set of LMIs without introducing any free-weighting matrices. The delay feedback controllers $\mathrm{H} 1$ and $\mathrm{H} 2$ designed can guarantee exponential synchronization of the complex dynamical network. Simulation results have been given to illustrate the effectiveness of the proposed method.

Competing interests

The authors declare that they have no competing interests.

Authors' contributions

All authors contributed equally to the writing of this paper. All authors read and approved the final manuscript.

\section{Author details}

${ }^{1}$ Department of Mathematics, Srinakharinwirot University, Sukhumvit 23 Road, Bangkok, 10110, Thailand. ${ }^{2}$ Centre of Excellence in Mathematics, CHE, Si Ayutthaya Road, Bangkok, 10400, Thailand. ${ }^{3}$ Department of Mathematics, Chiang Mai University, Huay Kaew Road, Chiang Mai, 50200, Thailand.

\section{Acknowledgements}

We would like to thank referees for their valuable comments and suggestions. This work is supported by the Thailand Research Fund (TRF), the Office of the Higher Education Commission (OHEC), Srinakharinwirot University (grant number MRG5580081), and the Centre of Excellence in Mathematics, the Commission on Higher Education, Thailand.

Received: 8 December 2013 Accepted: 22 April 2014 Published: 06 May 2014

\section{References}

1. Faloutsos, M, Faloutsos, P, Faloutsos, C: On power-law relationships of the Internet topology. Comput. Commun. Rev. 29, 251-263 (1999)

2. Albert, R, Jeong, H, Barabási, AL: Diameter of the world wide web. Nature 401, 130-131 (1999)

3. Williams, RJ, Martinez, ND: Simple rules yield complex food webs. Nature 404, 180-183 (2000)

4. Jeong, H, Tombor, B, Albert, R, Oltvai, Z, Barabási, AL: The large-scale organization of metabolic network. Nature 407, 651-653 (2000)

5. Wassrman, S, Faust, K: Social Network Analysis. Cambridge University Press, Cambridge (1994)

6. Strogatz, SH: Exploring complex networks. Nature 410, 268-276 (2001)

7. Zhang, Q, Lu, J, Lu, J, Tse, CK: Adaptive feedback synchronization of a general complex dynamical network with delayed nodes. IEEE Trans. Circuits Syst. II 55, 183-187 (2008)

8. Li, C, Chen, G: Synchronization in general complex dynamical networks with coupling delays. Physica A 343, 263-278 (2004) 
9. Li, K, Guan, S, Gong, X, Lai, CH: Synchronization stability of general complex dynamical networks with time-varying delays. Phys. Lett. A 372, 7133-7139 (2008)

10. Liu, T, Zhao, J: Synchronization of complex switched delay dynamical networks with simultaneously diagonalizable coupling matrices. J. Control Theory Appl. 6, 351-356 (2008)

11. Wang, XF, Chen, G: Pinning control of scale-free dynamical networks. Physica A 310, $521-531$ (2002)

12. Wang, XF, Chen, G: Synchronization scale-free dynamical networks: robustness and fragility. IEEE Trans. Circuits Syst. I 49, 54-62 (2002)

13. Wang, XF, Chen, G: Synchronization in small-world dynamical networks. Int. J. Bifurc. Chaos 12, 187-192 (2002)

14. Gao, H, Lam, J, Chen, G: New criteria for synchronization stability of general complex dynamical networks with coupling delays. Phys. Lett. A 306, 263-273 (2006)

15. Zhou, J, Xiang, L, Liu, Z: Global synchronization in general complex delayed dynamical networks and its applications. Phys. Lett. A 385, 729-742 (2007)

16. Wang, L, Dai, HP, Sun, YX: Synchronization criteria for a generalized complex delayed dynamical network model. Phys. Lett. A 383, 703-713 (2007)

17. Li, P, Yi, Z: Synchronization analysis of delayed complex networks with time-varying couplings. Phys. Lett. A 387 3729-3737 (2008)

18. Yue, D, Li, H: Synchronization stability of continuous/discrete complex dynamical networks with interval time-varying delays. Neurocomputing 73, 809-819 (2010)

19. Gu, K, Kharitonov, VL, Chen, J: Stability of Time-Delay System. Birkhäuser, Boston (2003)

20. Han, QL: Robust stability for a class of linear systems with time varying delay and nonlinear perturbation. Comput. Math. Appl. 47, 1201-1209 (2004)

21. Han, QL, Gu, K: Stability of linear systems with time-varying delay: a generalized discretized Lyapunov functional approach. Asian J. Control 3, 170-180 (2001)

22. Jiang, X, Han, QL: On $H_{\infty}$ control for linear systems with interval time-varying delay. Automatica 41, 2099-2106 (2005)

23. Park, P: A delay-dependent stability criterion for systems with uncertain time-invariant delays. IEEE Trans. Autom. Control 44, 876-877 (1999)

24. Shao, HY: New delay-dependent stability criteria for systems with interval delay. Automatica 45, 744-749 (2009)

25. Xu, S, Lam, J, Zou, Y: Further results on delay-dependent robust stability conditions of uncertain neutral systems. Int. J. Robust Nonlinear Control 15, 233-246 (2005)

26. Gu, K, Niculescu, Sl: Additional dynamics in transformed time-delay systems. IEEE Trans. Autom. Control 45, 572-575 (2000)

27. Han, QL: A descriptor system approach to robust stability of uncertain neutral systems with discrete and distributed delays. Automatica 40, 1791-1796 (2004)

28. Peng, C, Tian, YC: Delay-dependent robust stability criteria for uncertain systems with interval time-varying delay. J. Comput. Appl. Math. 214, 480-494 (2008)

29. Tian, J, Xiong, L, Liu, J, Xie, X: Novel delay-dependent robust stability criteria for uncertain neutral systems with time-varying delay. Chaos Solitons Fractals 40, 1858-1866 (2009)

30. Huang, T, Li, C: Chaotic synchronization by the intermittent feedback method. J. Comput. Appl. Math. 234, 1097-1104 (2010)

31. Xia, W, Cao, J: Pinning synchronization of delayed dynamical networks via periodically intermittent control. Chaos 19 $013120(2009)$

32. Cai, S, Liu, Z, Xu, F, Shen, J: Periodically intermittent controlling complex dynamical networks with time-varying delays to a desired orbit. Phys. Lett. A 373, 3846-3854 (2009)

33. Cai, S, Hao, J, He, Q, Liu, Z: Exponential synchronization of complex delayed dynamical networks via pinning periodically intermittent control. Phys. Lett. A 375, 1965-1971 (2011)

34. Cai, S, He, Q, Hao, J, Liu, Z: Exponential synchronization of complex networks with nonidentical time-delayed dynamical nodes. Phys. Lett. A 374, 2539-2550 (2010)

35. Yang, X, Cao, J: Stochastic synchronization of coupled neural networks with intermittent control. Phys. Lett. A 373 3259-3272 (2009)

36. Wang, Y, Hao, J, Zuo, Z: A new method for exponential synchronization of chaotic delayed systems via intermittent control. Phys. Lett. A 374, 2024-2029 (2010)

37. Zhang, W, Huang, J, Wei, P: Weak synchronization of chaotic neural networks with parameter mismatch via periodically intermittent control. Appl. Math. Model. 35, 612-620 (2011)

38. Yu, J, Hu, C, Jiang, H, Teng, Z: Exponential synchronization of Cohen-Grossberg neural networks via periodically intermittent control. Neurocomputing 74, 1776-1782 (2011)

39. Zhu, H, Cui, B: Stabilization and synchronization of chaotic systems via intermittent control. Commun. Nonlinear Sci. Numer. Simul. 15, 3577-3586 (2010)

40. Zhang, G, Lin, X, Zhang, X: Exponential stabilization of neutral-type neural networks with mixed interval time-varying delays by intermittent control. Circuits Syst. Signal Process. 33, 371-391 (2014)

41. Huang, J, Li, C, Han, G: Stabilization of delayed chaotic neural networks by periodically intermittent control. Circuits Syst. Signal Process. 28, 567-579 (2009)

42. Horn, RA, Johnson, CR: Matrix Analysis. Cambridge University Press, Cambridge (1985)

43. Botmart, T, Niamsup, P: Adaptive control and synchronization of the perturbed Chua system. Math. Comput. Simul. 75, 37-55 (2007)

10.1186/1687-1847-2014-116

Cite this article as: Botmart and Niamsup: Exponential synchronization of complex dynamical network with mixed time-varying and hybrid coupling delays via intermittent control. Advances in Difference Equations 2014, 2014:116 